\title{
On the Quotients of Cubic Hecke Algebras
}

\section{Louis Funar ${ }^{\star}$}

Institute of Mathematics, Bucharest, Romania, and Université de Paris-Sud, Orsay, France and (permanent address) UA 188 CNRS, Institut Fourier, BP 74, Mathèmatiques, Univ. Grenoble I, F-38402 Saint-Martin-D’Hères Cedex, France. E-mail: funar@fourier.grenet.fr

Received: 17 November 1993/in revised form: 3 January 1995

\begin{abstract}
Between the rank 3 quotients of cubic Hecke algebras there is essentially one of maximal dimension. We prove it has a unique Markov trace having values in a torsion module. Therefore the description of a Markov trace on the cubic Hecke algebra corresponding to $x^{3}+1$ and having the parameters $(1,1)$ is derived. Thus we obtain a numerical link invariant of finite degree, and define a whole sequence of $3^{\text {rd }}$ order Vassiliev invariants.
\end{abstract}

\section{Contents}

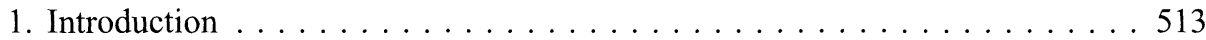

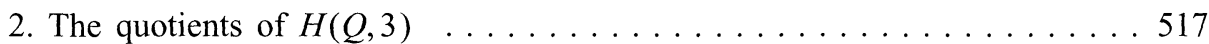

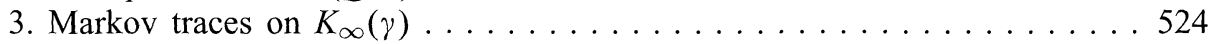

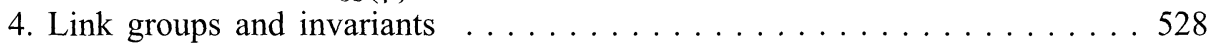

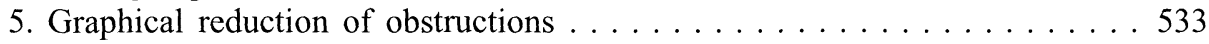

A. Appendix: The module $H(Q, 3) \ldots \ldots \ldots \ldots \ldots$. . . . . . . . . . . 549

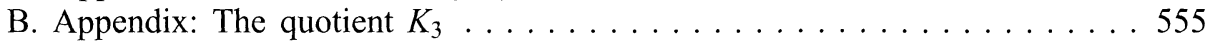

\section{Introduction}

The aim of this paper is to begin a systematic study of cubic Hecke algebras by analogy with the analysis carried out by Vaughan Jones (see [Jon87]) in the classical case of Hecke algebras. The motivation is to derive link invariants and Markov traces on the group algebra of the braid group.

We recall that Artin's braid group $B_{n}$ in $n$ strings is presented usually as

$$
\begin{gathered}
B_{n}=\left\langle b_{1}, b_{2}, \ldots, b_{n-1}\left|b_{l} b_{j}=b_{j} b_{l},\right| i-j\right|>1, i, j=1, n-1 ; \\
\left.b_{i+1} b_{l} b_{l+1}=b_{l} b_{l+1} b_{l}, \quad i=1, n-2\right\rangle .
\end{gathered}
$$

\footnotetext{
* Most of this work was done when the author prepared his PhD thesis at University of Paris-Sud and was partially supported by a BGF grant.
} 
and we have a sequence of natural inclusions

$$
B_{2} \subset B_{3} \subset B_{4} \subset \cdots \subset B_{n} \subset B_{n+1} \subset \cdots .
$$

On the other hand $B_{n}$ is the group of isotopy classes of $n$ strings lying in $\mathbf{R}^{2} \times[0,1]$ having nowhere horizontal tangent vectors, and fixed endpoints. Assume that the braid $x \in B_{n}$ lies in a box $[0,1] \times[0,1] \times[0,1]$ and we choose $n$ large, disjoint (and unknotted) circles outside the box which connect the pairs of upper and bottom endpoints. Artin's closure of $x$ is the link obtained this way, naturally oriented by choosing the up-down orientation for the strings in the box. Then every oriented link is Artin's closure $\hat{x}$ of some braid $x$. Moreover two links $\hat{x}$ and $\hat{y}$ (where $x, y \in \bigcup_{n} B_{n}$ ) are isotopic if and only if they are equivalent under a sequence of Markov moves consisting in:

1. Replacing $z \in B_{n}$ by a conjugate $c z c^{-1} \in B_{n}$.

2. Replacing $z \in B_{n}$ by $z b_{n}^{\varepsilon} \in B_{n+1}, \varepsilon \in\{-1,1\}$, or conversely.

Recall that a Markov trace on the group algebra of the braid group is a functional $t$ satisfying

1. $t(x y)=t(y x)$, if $x, y \in B_{n}$,

2. $t\left(x b_{n}\right)=z t(x), t\left(x b_{n}^{-1}\right)=\bar{z} t(x)$ if $x \in B_{n}$, with $z, \bar{z} \in \mathbf{C}^{*}$, called parameters.

Therefore the Markov traces, properly normalized, induce link invariants (see Sect. 4).

The usual Hecke algebras $H(q, n)$ are quotients

$$
H(q, n)=\mathbf{C}\left[B_{n}\right] /\left(b_{t}^{2}-(q-1) b_{i}-q, i=1, n-1\right), \quad q \in \mathbf{C}^{*} .
$$

The structure of these algebras is well-known (see [Bou82] and $H(q, n)$ are finitedimensional $\mathbf{C}[q]$-modules of dimension $n !$. The existence and the uniqueness of the Markov traces on the Hecke algebras lead to the famous Jones polynomial.

Definition 1.1. The generalized Hecke algebras are defined as the quotients

$$
H(Q, n)=\mathbf{C}\left[B_{n}\right] /\left(Q\left(b_{j}\right) ; j=1, n-1\right),
$$

for some polynomial $Q$, having $Q(0) \neq 0$. If the degree of $Q$ equals 3 we call them cubic Hecke algebras.

A natural question is to investigate the structure of these algebras and the Markov traces they support. In the general case we notice that some new features arise. In particular:

$$
\operatorname{dim}_{\mathbf{C}} H(Q, n)=\infty \quad \text { if } \operatorname{deg}(Q)>6 \text {, and } n \geqq 3 .
$$

Also even in the case of a cubic polynomial we have:

$$
\operatorname{dim}_{\mathbf{C}} H(Q, n)=\infty \text { if } n>6, \operatorname{deg}(Q)=3 .
$$

We shall be concerned in this paper with the (tower of) cubic Hecke algebras obtained for a cubic polynomial $Q$, having $Q(0) \neq 0$.

The cubic Hecke algebra $H(Q, n)$ surjects onto the ordinary quadratic algebras $H(P, n)$, (for every quadratic polynomial $P$ dividing $Q$ ) and also onto the BirmanWenzl ([BW89]) algebra. Hence we can lift the Markov traces on the last ones 
to get generically four families of Markov traces on $H(Q, *)$. Their parameters should satisfy some algebraic equations. The first question which arises naturally is whenever some other Markov traces exists. We shall give a partial positive answer to this question by constructing one such, for a fixed polynomial $Q$, and special values of parameters. We call a Markov trace multiplicative if the associated link invariant behaves multiplicatively with respect to the connected sum of links. Set $Q_{\gamma}=X^{3}-\gamma$. Our main result can be stated as (see $4.1,4.3$ ):

Main Theorem 1.2. There exists a multiplicative Markov trace with parameters $(1,1)$ on $H\left(Q_{-1}, *\right)$, whose associated link invariant $F$ is algorithmically computable, takes only the values -1 and 1 and is not a Vassiliev invariant of finite type.

We remark that this Markov trace cannot be a lift of the Jones-Ocneanu trace on the (quadratic) Hecke algebras since the parameters would be $\left(1,-1+2 \exp \left(\frac{\pi i}{3}\right)\right)$ or $\left(1,-1-2 \exp \left(\frac{\pi l}{3}\right)\right)$. From 2.11 it cannot be a lift of the Birman-Wenzl trace either.

We shall outline below the strategy of our proof.

We introduce the quotient $K_{n}(\gamma)=H\left(Q_{\gamma}, n\right) / I_{n}$, where the two-sided ideal $I_{n}$ is generated by

$$
\begin{aligned}
& b_{l+1} b_{l}^{2} b_{l+1}+b_{i} b_{i+1}^{2} b_{l}+b_{l}^{2} b_{l+1} b_{l}+b_{i} b_{l+1} b_{i}^{2}+b_{i}^{2} b_{l+1}^{2} \\
& \quad+b_{i+1}^{2} b_{i}^{2}+\gamma b_{i}+\gamma b_{i+1}, \quad i=1, n-2 .
\end{aligned}
$$

Then $K_{n}(\gamma)$ are finite dimensional modules and we are able to describe all Markov traces they support (see 3.4 for a more precise statement):

Theorem on the Quotient Trace 1.3. There exists an unique Markov trace on $K_{*}(-1)$ whose parameters must satisfy $z^{3}=1, z=z^{2}$ and takes values in $\mathbf{Z} / 6 \mathbf{Z}$.

Dually we computed the link group (see 3.1) associated to $K_{\infty}(\gamma)$ and the two parameters $(z, \bar{z})$. It is (3.4), a cyclic torsion group of order 6 or it vanishes. Roughly speaking the link group of a quotient is the group generated by the isotopy classes of oriented links modulo the skein relations dictated by the generators of the ideals $I_{n}$. So it turns out that these various link groups are not always torsion free.

Idea of Proof. It is simple to check that the parameters have to be these ones and the Markov trace on $K_{*}(-1)$, if either exists, is uniquely defined. For the existence part we restrict first by checking that the functional $t$ satisfying only the recursive conditions

$$
t\left(x b_{n}\right)=z t(x), \quad t\left(x b_{n}^{-1}\right)=\bar{z} t(x) \text { if } x \in K_{n}(-1),
$$

(which we call admissible functional), is well-defined.

The method of proof is greatly inspired from [Ber78] and is given in Sect. 5. We define a huge graph whose vertices are the elements of the abelian semi-group associated to the free group in $n-1$ letters (in the first instance) and whose edges correspond to elements which differ by exactly one relation (from the set of relations defining $K_{n}(\gamma)$ ).

If we used our relations in only one direction (i.e. we may replace $a$ by $b$ but not $b$ by $a$ ) we would arrive at orienting the edges of this graph, and we may ask 
whenever the minimal elements of each connected component of the graph exist and are unique. This will provide a basis for $K_{n}(\gamma)$ if sufficiently many relations are added in order to obtain the uniqueness. For the existence of minimal elements the usual procedure is to use the lexicographic order on the free group on $n-1$ letters and to replace always a word by smaller ones. We have carried out this algorithm for $H(Q, 3)$ in Appendix $\mathrm{A}$ and we can see the technical difficulties which may be encountered. So having yet in mind a certain reduction process, we define the oriented edges as follows: exactly one monomial may be changed using one of the rules

$$
\begin{aligned}
& \text { (C0)(j) } A b_{j}^{3} B \rightarrow A B, \\
& \text { (C1)(j) } A b_{j+1} b_{j} b_{j+1} B \rightarrow A b_{j} b_{J+1} b_{j} B, \\
& \text { (C2)(j) } A b_{j+1} b_{j}^{2} b_{j+1} B \rightarrow A S_{j} B, \\
& \text { (C12)(j) } A b_{j+1} b_{j}^{2} b_{j+1}^{2} B \rightarrow A b_{j}^{2} b_{j+1}^{2} b_{j} B, \\
& \text { (C21)(j) } A b_{j+1}^{2} b_{j}^{2} b_{j+1} B \rightarrow A b_{j} b_{j+1}^{2} b_{j}^{2} B .
\end{aligned}
$$

Also some unoriented edges must be added. They correspond to a change in a monomial of type

$$
\left(P_{l j}\right) A b_{i} b_{j} B \rightarrow A b_{j} b_{l} B \quad \text { whenever }|i-j|>1 .
$$

We remark that we were forced to add some relations (knowing that they hold already in $H(Q, n))$ which make the reduction process ambiguous. The reason is to assure the existence of descending paths to some minimal points even if closed oriented loops may be found in the graph. And we shall check the existence and uniqueness of minimal elements up to unoriented paths in this semi-oriented graph by means of the so-called Pentagon Lemma 5.3. When this approach is not successful we shall widen our graph to a tower of graphs modeling not $K_{n}(\gamma)$ but the functionals on $K_{n}(\gamma)$ satisfying a recurrent condition which permits to reduce further the minimal elements. Here the Colored Pentagon Lemma 5.6 (in fact a variant of 5.3) can be applied and the problem is reduced to some algebraic computations. This shows that the main obstructions lie in $K_{4}(\gamma)$ not in $K_{3}(\gamma)$, as it could be expected from the study of quadratic Hecke algebras. When we wish to check if the commutativity condition for the functional is actually a Markov trace another obstruction appears in $K_{4}(\gamma)$. This explains why torsion arises in the link group and ends the proof of Theorem 1.3.

We come back to the cubic Hecke algebras considered above. In the fourth section we prove that the Markov trace we constructed on $K_{\infty}(\gamma)$, and taking values in $\mathbf{Z} / 6 \mathbf{Z}$, has a lift (as the multiplicative Markov trace) to $H\left(Q_{-1}, \infty\right)$ which is integer valued. A link invariant $F$, which is not a Vassiliev invariant of finite degree, is derived in this manner.

We define further a whole sequence of $3^{\text {rd }}$ order Vassiliev invariants, which in degree 0 correspond to $F$, and are algorithmically computable, using the method of Baez [Bae92]. Whenever some of them are really new (so they are not limits of classical Vassiliev invariants) we don't know at this moment.

The existence of a deformation of the homogeneous quotient $K_{*}(\gamma)$ (see 2.13) enables us to believe that our main result can be established in more generality for an arbitrary cubic polynomial $Q$ (for some precise values of the parameters), using the same method of computing the obstructions. However the explicit computations are rather cumbersome. 


\section{The Quotients of $H(Q, 3)$}

The generalized Hecke algebras were introduced by analogy with the classical case as the quotients

$$
H(Q, n)=\mathbf{C}\left[B_{n}\right] /\left(Q\left(b_{j}\right) ; j=1, n-1\right)
$$

of the group algebra of the braid group by the ideal generated by $Q\left(b_{j}\right)$, where $Q$ is a polynomial having $Q(0) \neq 0$. We wish first to consider the quotients $P(3)$ of $H(Q, 3)$. We need therefore to know $\operatorname{dim}_{\mathbf{C}}(Q, 3)$.

Proposition 2.1. For all cubic polynomials $Q$ with $Q(0) \neq 0$, we have

$$
\operatorname{dim}_{\mathbf{C}} H(Q, 3)=24 \text {. }
$$

Proof. Since $Q(0) \neq 0$ we may restrict to the case when the exponents of the $b_{i}$ 's are 0,1 or 2 . Set $w_{n, k}=b_{n} b_{n-1} \ldots b_{k+1} b_{k}^{2} b_{k+1} \ldots b_{n} \in H(Q, n+1)$ and $Q=X^{3}-$ $\alpha x^{2}-\beta x-\gamma$.

Lemma 2.2. Set $r_{n, j}=b_{j} b_{j+1} \ldots b_{n-1}$. The following commutation rules hold in $H(Q, n+1)$ :

$$
\begin{aligned}
& b_{i} w_{n, j}=w_{n, j} b_{l} \text { if } i \neq j-1 \text { and } i<n, \\
& b_{n} w_{n, j}=\alpha w_{n, j}+\beta w_{n-1, j} b_{n}+\gamma r_{n, j} w_{n, j+1} r_{n, j}^{-1}, \\
& w_{n, j} b_{n}=\alpha w_{n, j}+\beta b_{n} w_{n-1, j}+\gamma r_{n, j}^{-1} w_{n, j+1} r_{n, j},
\end{aligned}
$$

where $w_{n, n}=b_{n}^{2}$.

Proof of Lemma. If $i<j-1$ we obtain the first relation since the $b_{k}$ 's involved in $w_{n, j}$ have $|k-i| \geqq 2$. Now

$$
\begin{aligned}
b_{n-1} w_{n, j} & =\left(b_{n-1} b_{n} b_{n-1}\right) \ldots b_{J}^{2} \ldots b_{n-1} b_{n} \\
& =b_{n} b_{n-1} b_{n} \ldots b_{J}^{2} \ldots b_{n-1} b_{n} \\
& =b_{n} b_{n-1} b_{n-2} \ldots b_{J}^{2} \ldots b_{n-2}\left(b_{n} b_{n-1} b_{n}\right) \\
& =b_{n} b_{n-1} b_{n-2} \ldots b_{J}^{2} \ldots b_{n-2} b_{n-1} b_{n} b_{n-1} \\
& =w_{n, j} b_{n-1}
\end{aligned}
$$

proving the first relation for $i=n-1$. Similarly for all $i \geqq j$ we have

$$
\begin{aligned}
b_{i} w_{n, j} & =b_{l} b_{n} b_{n-1} \ldots b_{l+2} b_{i+1} b_{i} b_{i-1} \ldots b_{j}^{2} \ldots b_{n} \\
& =b_{n} b_{n-1} \ldots b_{i+2}\left(b_{l} b_{l+1} b_{l}\right) b_{i-1} \ldots b_{j}^{2} \ldots b_{n} \\
& =b_{n} b_{n-1} \ldots b_{i+2} b_{l+1} b_{i} b_{i+1} b_{i-1} \ldots b_{j}^{2} \ldots b_{i-1} b_{l} b_{l+1} \ldots b_{n} \\
& =b_{n} b_{n-1} \ldots b_{i+2} b_{l+1} b_{i} b_{i-1} \ldots b_{J}^{2} \ldots b_{i-1}\left(b_{i+1} b_{i} b_{i+1}\right) \ldots b_{n} \\
& =b_{n} b_{n-1} \ldots b_{j}^{2} \ldots b_{l-1} b_{l} b_{l+1} b_{l} \ldots b_{n} \\
& =w_{n, j} b_{i} .
\end{aligned}
$$

So the first commutation relations are proved. 
Sublemma 2.3. $w_{n, j} b_{n}=b_{n-1}^{-1} b_{n} w_{n-1, j} b_{n-1} b_{n} b_{n-1}$ for $j=1, n-1$.

Set first $j<n-1$. We shall use $b_{n-1} b_{n}=b_{n} b_{n-1} b_{n} b_{n-1}^{-1}$ in what follows:

$$
\begin{aligned}
w_{n, j} b_{n}= & b_{n} b_{n-1} \ldots b_{j}^{2} \ldots b_{n-1} b_{n}^{2}=b_{n} b_{n-1} \ldots b_{j}^{2} \ldots b_{n-2} b_{n} b_{n-1} b_{n} b_{n-1}^{-1} b_{n} \\
= & \left(b_{n} b_{n-1} b_{n}\right) b_{n-2} \ldots b_{j}^{2} \ldots b_{n-2} b_{n-1} b_{n} b_{n-1}^{-1} b_{n} \\
= & b_{n-1} b_{n} b_{n-1} b_{n-2} \ldots b_{j}^{2} \ldots b_{n-2} b_{n-1} b_{n}\left(\gamma^{-1} b_{n-1}^{2}-\alpha \gamma^{-1} b_{n-1}-\beta \gamma^{-1}\right) b_{n} \\
= & \gamma^{-1} b_{n-1} b_{n} b_{n-1} b_{n-2} \ldots b_{j}^{2} \ldots b_{n-2}\left(b_{n-1} b_{n} b_{n-1}\right) b_{n-1} b_{n} \\
& -\alpha \gamma^{-1} b_{n-1} b_{n} b_{n-1} b_{n-2} \ldots b_{j}^{2} \ldots b_{n-2} b_{n-1} b_{n} b_{n-1} b_{n} \\
& -\beta \gamma^{-1} b_{n-1}\left(b_{n} b_{n-1} b_{n-2} \ldots b_{j}^{2} \ldots b_{n-2} b_{n-1} b_{n}\right) b_{n} .
\end{aligned}
$$

Using also the previous commutation rules for $i=n-1$ we obtain

$$
\begin{aligned}
w_{n, j} b_{n}= & \gamma^{-1} b_{n-1} b_{n} b_{n-1} b_{n-2} \ldots b_{j}^{2} \ldots b_{n-2} b_{n} b_{n-1}\left(b_{n} b_{n-1} b_{n}\right) \\
& -\alpha \gamma^{-1} b_{n-1} b_{n} b_{n-1} b_{n-2} \ldots b_{J}^{2} \ldots b_{n-2} b_{n-1}^{2} b_{n} b_{n-1} \\
& -\beta \gamma^{-1} b_{n} b_{n-1} b_{n-2} \ldots b_{j}^{2} \ldots b_{n-2} b_{n-1}\left(b_{n} b_{n-1} b_{n}\right) \\
= & \gamma^{-1} b_{n-1}\left(b_{n} b_{n-1} b_{n}\right) b_{n-2} \ldots b_{J}^{2} \ldots b_{n-2} b_{n-1}^{2} b_{n} b_{n-1} \\
& -\alpha \gamma^{-1} b_{n-1}\left[b_{n} w_{n-1, j} b_{n-1}\right] b_{n} b_{n-1}-\beta \gamma^{-1}\left[b_{n} w_{n-1, j} b_{n-1}\right] b_{n} b_{n-1} \\
= & \left(\gamma^{-1} b_{n-1}^{2}-\alpha \gamma^{-1} b_{n-1}-\beta \gamma^{-1}\right)\left[b_{n} w_{n-1, j} b_{n-1}\right] b_{n} b_{n-1} \\
= & b_{n-1}^{-1} b_{n} w_{n-1, j} b_{n-1} b_{n} b_{n-1} .
\end{aligned}
$$

For $j=n-1$ we may write in the same manner:

$$
\begin{aligned}
b_{J+1} b_{j}^{2} b_{j+1}^{2}= & b_{j+1} b_{j}\left(b_{J+1} b_{J} b_{J+1} b_{J}^{-1}\right) b_{j+1}=b_{J} b_{j+1} b_{J}^{2} b_{j+1} b_{j}^{-1} b_{j+1} \\
= & \gamma^{-1} b_{j} b_{j+1} b_{j}^{2} b_{j+1} b_{j}^{2} b_{j+1}-\alpha \gamma^{-1} b_{j} b_{j+1} b_{j}^{2} b_{j+1} b_{j} b_{j+1} \\
& -\beta \gamma^{-1} b_{j} b_{j+1} b_{j}^{2} b_{j+1}^{2}=\gamma^{-1} b_{j} b_{j+1} b_{j} b_{j+1} b_{j} b_{J+1} b_{j} b_{j+1} \\
& -\alpha \gamma^{-1} b_{J} b_{j+1} b_{j}^{3} b_{J+1} b_{j}-\beta \gamma^{-1} b_{J+1} b_{j} b_{j+1} b_{J} b_{J+1}^{2} \\
= & \gamma^{-1} b_{j}^{2} b_{J+1} b_{J}^{3} b_{j+1} b_{J}-\alpha \gamma^{-1} b_{j} b_{J+1} b_{J}^{3} b_{j+1} b_{j}-\beta \gamma^{-1} b_{J+1} \\
& \times b_{j}^{2} b_{J+1} b_{J} b_{j+1}=\left(\gamma^{-1} b_{J}^{2}-\alpha \gamma^{-1} b_{J}-\beta \gamma^{-1}\right) b_{j+1} \\
& \times b_{j}^{3} b_{J+1} b_{J}=b_{j}^{-1} b_{J+1} b_{j}^{3} b_{j+1} b_{J},
\end{aligned}
$$

which ends the proof of the sublemma. 
We are able now to prove our lemma. From above we have:

$$
\begin{aligned}
w_{n, j} b_{n} & =b_{n-1}^{-1} b_{n}\left(w_{n-1, j} b_{n-1}\right) b_{n} b_{n-1} \\
& =b_{n-1}^{-1} b_{n} b_{n-2}^{-1} b_{n-1}\left(w_{n-2, j} b_{n-2}\right) b_{n-1} b_{n-2} b_{n} b_{n-1} \\
& =\cdots=r_{n, j+1}^{-1}\left[b_{n} b_{n-1} \ldots b_{j+2}\left(w_{j+1, j} b_{j+1}\right) b_{j+2} \ldots b_{n}\right] r_{n, j+1} .
\end{aligned}
$$

We denote $b_{n} \ldots b_{J+2}=s$ and $b_{j+2} \ldots b_{n}=t$ for simplicity. Thus

$$
\begin{aligned}
w_{n, j} b_{n}= & r_{n, j+1}^{-1} s\left(b_{j}^{-1} b_{j+1} b_{j}^{3} b_{j+1} b_{J}\right) \operatorname{tr}_{n, j+1}=\alpha r_{n, j+1}^{-1} s\left(b_{j}^{-1} b_{j+1} b_{j}^{2} b_{j+1} b_{j}\right) \operatorname{tr}_{n, j+1} \\
& +\beta r_{n, j+1}^{-1} s\left(b_{j}^{-1} b_{j+1} b_{j} b_{J+1} b_{J}\right) \operatorname{tr}_{n, j+1}+\gamma r_{n, j+1}^{-1} s\left(b_{j}^{-1} b_{j+1}^{2} b_{j}\right) \operatorname{tr}_{n, j+1} \\
= & \alpha r_{n, j}^{-1} s b_{j+1} b_{j}^{2} b_{j+1} \operatorname{tr}_{n, j}+\beta y+\gamma r_{n, j}^{-1} w_{n, j+1} r_{n, j},
\end{aligned}
$$

where we met

$$
\begin{aligned}
y= & r_{n, j}^{-1}\left(b_{n} \ldots b_{j+1} b_{j} b_{J+1} \ldots b_{n}\right) r_{n, J}=r_{n, j}^{-1}\left(b_{n} \ldots b_{j} b_{J+1} b_{j} \ldots b_{n}\right) r_{n, j} \\
= & r_{n, j}^{-1} b_{j}\left(b_{n} \ldots b_{j+2} b_{j+1} b_{J+2} \ldots b_{n}\right) b_{j} r_{n, j}=\cdots=r_{n, j}^{-1}\left(b_{j} b_{J+1} \ldots b_{n-2}\right. \\
& \left.\times\left(b_{n} b_{n-1} b_{n}\right) b_{n-2} \ldots b_{j}\right) r_{n, j}=b_{n} w_{n-1 . J},
\end{aligned}
$$

and this proves that

$$
w_{n, j} b_{n}=\alpha r_{n, j}^{-1} w_{n, j} r_{n, j}+\beta b_{n} w_{n-1, j}+\gamma r_{n, j}^{-1} w_{n, j+1} r_{n, j} .
$$

But $r_{n, j} w_{n, j}=w_{n, j} r_{n, j}$ according to the first commutation rule, so we are done.

For $n=2$ the relations of the lemma read:

$$
\begin{aligned}
b_{2} b_{1}^{2} b_{2} b_{1} & =b_{1} b_{2} b_{1}^{2} b_{2}, \\
b_{2}^{2} b_{1}^{2} b_{2} & =b_{1} b_{2}^{2} b_{1}^{2}+\alpha\left(b_{2} b_{1}^{2} b_{2}-b_{1} b_{2}^{2} b_{1}\right)+\beta\left(b_{1}^{2} b_{2}-b_{1} b_{2}^{2}\right) \\
b_{2} b_{1}^{2} b_{2}^{2} & =b_{1}^{2} b_{2}^{2} b_{1}+\alpha\left(b_{2} b_{1}^{2} b_{2}-b_{1} b_{2}^{2} b_{1}\right)+\beta\left(b_{2} b_{1}^{2}-b_{2}^{2} b_{1}\right) .
\end{aligned}
$$

Lemma 2.4. Any word $w$ in $b_{1}$ and $b_{2}$ is equivalent (as an element of $H(Q, 3)$ ) to a sum of words having the degree in $b_{2}$ at most 2.

Proof. In fact if the degree in $b_{2}$ is at least 3 then the word contains one of the monomials $b_{2}^{a} b_{1}^{b} b_{2}^{c}$ with $a+c \geqq 3$ or $b_{2} b_{1}^{b} b_{2} b_{1}^{c} b_{2}$. We prove that in both situations the degree may be reduced.

In the first case if $b=1$ then we replace $b_{2} b_{1} b_{2}$ by $b_{1} b_{2} b_{1}$. If $b=2$ then $a$ or $c$ equals 2 so we can apply one of the above written relations.

In the second case if $b$ or $c$ equals 1 again we may replace $b_{2} b_{1} b_{2}$ by $b_{1} b_{2} b_{1}$. If $b=c=2$ then

$$
b_{2} b_{1}^{2} b_{2} b_{1}^{2} b_{2}=b_{1} b_{2} b_{1}^{2} b_{1} b_{2}=b_{1}^{2} b_{2} b_{1}^{2} b_{2}^{2}
$$

so the third relation may be used to reduce the degree of $w$, thus proving our claim. 
It follows that the following elements generate the vector space $H(Q, 3)$ :

$$
\begin{aligned}
& e_{1}=1, e_{2}=b_{1}, e_{3}=b_{1}^{2}, e_{4}=b_{2}, e_{5}=b_{2}^{2}, e_{6}=b_{1} b_{2}, e_{7}=b_{2} b_{1}, e_{8}=b_{1}^{2} b_{2}, \\
& e_{9}=b_{2} b_{1}^{2}, e_{10}=b_{1} b_{2}^{2}, e_{11}=b_{2}^{2} b_{1}, e_{12}=b_{1}^{2} b_{2}^{2}, e_{13}=b_{2}^{2} b_{1}^{2}, e_{14}=b_{1} b_{2} b_{1}, \\
& e_{15}=b_{1}^{2} b_{2} b_{1}, e_{16}=b_{1} b_{2} b_{1}^{2}, e_{17}=b_{1} b_{2}^{2} b_{1}^{2}, e_{18}=b_{1}^{2} b_{2} b_{1}^{2}, e_{19}=b_{1}^{2} b_{2}^{2} b_{1}, \\
& e_{20}=b_{1} b_{2}^{2} b_{1}, e_{21}=b_{1}^{2} b_{2}^{2} b_{1}^{2}, e_{22}=b_{2} b_{1}^{2} b_{2}, e_{23}=b_{2} b_{1}^{2} b_{2} b_{1}=b_{1} b_{2} b_{1}^{2} b_{2}, \\
& e_{24}=b_{2} b_{1}^{2} b_{2} b_{1}^{2}=b_{1} b_{2} b_{1}^{2} b_{2} b_{1}=b_{1}^{2} b_{2} b_{1}^{2} b_{2} .
\end{aligned}
$$

We remark that for $\alpha=\beta=0, \gamma=1$, so $Q_{1}=X^{3}-1$, the algebra $H\left(Q_{1}, 3\right)$ is the group algebra of a group of order 24 . In fact $\left\{e_{1}, e_{2}, \ldots, e_{24}\right\}$ becomes a group in which the multiplication law is induced by the following identities:

$$
b_{2}^{2} b_{1}^{2} b_{2}=b_{1} b_{2}^{2} b_{1}^{2} ; b_{2} b_{1}^{2} b_{2}^{2}=b_{1}^{2} b_{2}^{2} b_{1} ; b_{2} b_{1}^{2} b_{2} b_{1}=b_{1} b_{2} b_{1}^{2} b_{2} \text {. }
$$

It follows that $H\left(Q_{1}, 3\right)$ is a semi-simple algebra, hence for $Q$ generic and sufficiently close to $Q_{1}$ the algebra $H(Q, 3)$ will be also a semi-simple algebra of the same dimension. This ends the proof of the proposition for generic $Q$ close to $Q_{1}$. The complete proof for all $Q$ is given in Appendix A.

Remember that the Markov trace on the quadratic Hecke algebras (which is unique [Jon87]) has the following multiplicative property:

$$
\operatorname{tr}\left(x b_{n}\right)=\operatorname{tr}(x) \operatorname{tr}\left(b_{n}\right), \quad \text { when } x \in H(Q, n),
$$

which implies that:

$$
\operatorname{tr}(x y)=\operatorname{tr}(x) \operatorname{tr}(y), \quad \text { when } x \in H(Q, n), y \in\left\langle 1, b_{n}, b_{n+1}, \ldots, b_{n+k}\right\rangle .
$$

However we cannot expect that this property will extend to higher level algebras and the Markov traces they support.

Definition 2.5. We say that a Markov trace $t$ is quasi-multiplicative if

$$
t\left(x b_{n}^{k}\right)=t(x) t\left(b_{n}^{k}\right)
$$

holds, when $x \in H(Q, n), k \in \mathbf{Z}$, and multiplicative if the stronger condition

$$
\operatorname{tr}(x y)=\operatorname{tr}(x) \operatorname{tr}(y) \text { when } x \in H(Q, n), y \in\left\langle 1, b_{n}, b_{n+1}, \ldots, b_{n+k}\right\rangle
$$

is verified.

Lemma 2.6. In the case of cubic Hecke algebras the Markov traces are quasimultiplicative.

In fact we have $b_{n}^{2}=\alpha b_{n}+\beta+\gamma b_{n}^{-1}$. We derive then the multiplicativity for $k=2$, since for $k \in\{-1,0,1\}$ is already contained in the definition of the Markov traces. This will imply the quasi-multiplicative property for all $k$.

Notice that a general Markov trace on the cubic Hecke algebra need not be a multiplicative one. 
Set then $B$ for the base of $H(Q, 3)$ considered above. A general relation yielding a rank 3 quotient takes therefore the form:

$$
R(\mu): \sum_{x \in B} \mu_{x} x=0, \quad \text { where } \quad\left(\mu_{x}\right)_{x \in B} \in \mathbf{C}^{24} .
$$

Set $\omega=\left(\mu_{b_{2} b_{1}^{2} b_{2}}, \mu_{b_{1} b_{2} b_{1}^{2} b_{2}}, \mu_{b_{1}^{2} b_{2} b_{1}^{2} b_{2}}\right) \in \mathbf{C}^{3}$ and $M_{Q}=\left[\begin{array}{lll}0 & 1 & 0 \\ 0 & 0 & 1 \\ \gamma & \beta & \alpha\end{array}\right]$.

Let $\lambda_{i}, i=1,3$ be the eigenvalues of $M_{Q}$ and $E_{Q}=\left\{\left(1, \lambda_{l}, \lambda_{i}^{2}\right), i=1,3\right\}$ be the eigenvectors of $M_{Q}$. Observe that $\lambda_{i} \neq 0$ since $\gamma \neq 0$.

Consider now a proper quotient of $P(3)=H(Q, 3) / I_{3}$. We can define therefore a whole tower of quotients $P(n)=H(Q, n) / I_{n}$ by defining $I_{n}$ as the two-sided ideal of $H(Q, n)$ generated by $I_{3}$. We call the tower $P(*)$ the quotient determined by $P(3)$.

Proposition 2.7. Suppose that for some relation $R(\mu)$ holding in $P(3)$ the following (generic) condition:

(*) (the degree 2 polynomial having the vector of coefficients $\omega$ has no common roots with $Q)$ is fulfilled. Then for fixed $(z, t) \in \mathbf{C}^{* 2}$ there exists at most one Markov trace on $P(*)$ with parameters $(z, t)$.

Proof. Define recursively the modules $L_{n}$ by

$$
\begin{aligned}
L_{2} & =H(Q, 2), \\
L_{3} & =\mathbf{C}\left\langle b_{1}^{i} b_{2}^{j} b_{1}^{k} ; i, j, k \in\{0,1,2\}\right\rangle, \\
L_{n+1} & =\mathbf{C}\left\langle a b_{n}^{\varepsilon} b ; \varepsilon \in\{1,2\}\right\rangle \oplus L_{n} .
\end{aligned}
$$

Lemma 2.8. Under the natural projection $\pi$ on $P(n), L_{n}$ surjects onto $P(n)$.

Proof. For $n=2$ it is trivial. For $n=3$ we remark that

$$
\sum_{x \in B^{\prime}} \omega_{x} x \in \pi\left(L_{3}\right)
$$

where we met $B^{\prime}=\left\{b_{2} b_{1}^{2} b_{2}, b_{1} b_{2} b_{1}^{2} b_{2}, b_{1}^{2} b_{2} b_{1}^{2} b_{2}\right\}$. But $L_{3}$ is $b_{1}$-invariant, so also

$$
\sum_{x \in B^{\prime}}\left(M_{Q} \omega\right)_{x} x=\sum_{x \in B^{\prime}} b_{1} x \in \pi\left(L_{3}\right) .
$$

The hypothesis implies $b_{2} b_{1}^{2} b_{2} \in \pi\left(L_{3}\right)$, and we are done.

Consider now $w \in P(n+1)$ represented by a word in the $b_{l}$ 's having only positive exponents. We assume the degree of the word in the variable $b_{n}$ is minimal among all words (with positive exponents) representing $w$.

If the degree is less or equal to 1 there is nothing to prove.

If the degree is 2 , then $w=u b_{n}^{2} v, u, v \in P(n)$, so using the induction hypothesis we are done, or else $w=u b_{n} z b_{n} v$, and $u, z, v \in P(n)$. So $z=x b_{n-1}^{\varepsilon} y$ with $x, y \in$ $P(n-1)$ by the induction and $\varepsilon \in\{0,1,2\}$. If $\varepsilon=0$ then $w$ may be reduced to $u z b_{n}^{2} v$. If $\varepsilon=1$ then $w=u b_{n} x b_{n-1} y b_{n} v=u x b_{n-1} b_{n} b_{n-1} y v$, hence the degree of $w$ 
may be reduced by 1 , contradiction. If $\varepsilon=2$ then $w=u x b_{n} b_{n-1}^{2} b_{n} y v$. But (from the definition of $P(n+1)$ ) we have

$$
b_{n} b_{n-1}^{2} b_{n} \in \mathbf{C}\left\langle b_{n-1}^{l} b_{n}^{l} b_{n-1}^{k} ; i, j, k=0,2\right\rangle,
$$

hence we reduced $w$ to a word of type $u^{\prime} b_{n}^{2} v^{\prime}$.

If the degree of $w$ is at least 3 we shall contradict the minimality. In fact $w$ contains a sub-word $w^{\prime}=b_{n}^{a} u b_{n}^{b}, u \in P(n)$ and $a+b \geqq 3$, or else a sub-word $w^{\prime \prime}=b_{n} u b_{n} v b_{n}, u, v \in P(n)$.

In the first case using the induction we can write $u=x b_{n-1}^{\varepsilon} y, x, y \in P(n-2)$.

If $\varepsilon=0$, then

$$
w^{\prime}=b_{n}^{a+b} x y=\alpha b_{n}^{a+b-1} x y+\beta b_{n}^{a+b-2} x y+\gamma b_{n}^{a+b-3} x y,
$$

hence its degree reduces by 1 .

If $\varepsilon=1$, then

$$
w^{\prime}=b_{n}^{a-1} x b_{n} b_{n-1} b_{n} y b_{n}^{b-1}=b_{n}^{a-1} x b_{n-1} b_{n} b_{n-1} y b_{n}^{b-1},
$$

again its degree is reduced by 1 .

If $\varepsilon=2$, then $a$ or $b$ equals 2 . Say $a=2$. We can write

$$
\begin{aligned}
w^{\prime}= & x b_{n}^{2} b_{n-1}^{2} b_{n} y b_{n}^{b-1}=x b_{1} b_{2}^{2} b_{1}^{2} y b_{n}^{b-1}+\alpha\left(b_{2} b_{1}^{2} b_{2}-b_{1} b_{2}^{2} b_{1}\right) y b_{n}^{b-1} \\
& +\beta\left(b_{1}^{2} b_{2}-b_{1} b_{2}^{2}\right) y b_{n}^{b-1}
\end{aligned}
$$

still contradicting the minimality of the degree of $w$.

In the second case we write also $u=x b_{n-1}^{\varepsilon} y, v=r b_{n-1}^{\delta} s$ with $x, y, r, s \in$ $P(n-1)$.

If $\varepsilon$ or $\delta$ equals 1 then, after some obvious commutation, the word $w^{\prime \prime}$ contains a factor $b_{2} b_{1} b_{2}$ which, when replaced by $b_{1} b_{2} b_{1}$ reduces the degree by 1 .

If $\varepsilon=\delta=2$, then

$$
w^{\prime \prime}=x b_{n} b_{n-1}^{2} b_{n} y r b_{n-1}^{2} b_{n} s .
$$

We use the homogeneity to replace $b_{n} b_{n-1}^{2} b_{n}$ by a sum of elements of type $b_{n-1}^{l} b_{n}^{j} b_{n-1}^{k}$. Each term of the expression of $w^{\prime \prime}$ which comes from a factor having $j<2$ has the degree less than it had before. The remaining terms are

$$
x b_{n-1}^{l} b_{n}^{2} b_{n-1}^{k} y r b_{n-1}^{2} b_{n} s
$$

so they contain a sub-word $b_{n}^{2} u b_{n}$ whose degree we already know may be reduced as above. This proves our claim. hence

Now the Markov traces $t$ on $H(Q, \infty)$ are quasi-multiplicative from Lemma 2.6,

$$
t\left(x b_{n}^{\varepsilon} y\right)=t\left(b_{n}^{\varepsilon}\right) t(y x),
$$

and $y x \in P(n)$. Therefore the extension of $t$, by recursion, from $P(n)$ to $P(n+1)$, if it ever exists, is unique. This ends the proof of our proposition.

Remark 2.9. We know that on $H(Q, \infty)$ there are at least 3 families of Markov traces whose parameters satisfy some linear conditions, coming from the Markov traces on quadratic Hecke algebras. In fact we have natural projections $H(Q, \infty) \rightarrow$ $H\left(Q_{i}, \infty\right)$, if $Q_{i}$ is a degree 2 factor of $Q$. 
We shall begin therefore to investigate the case when $\operatorname{dim}_{C} P(3)$ is maximal. According to 2.8 we have $\operatorname{dim}_{\mathbf{C}} P(3) \leqq 21$ for a generic quotient. We remark that we may always suppose $\gamma=1$ because we have an isomorphism of algebras $H(Q, n) \cong H\left(\gamma^{-1} Q, n\right)$. We shall assume that the hypothesis of Proposition 2.7 is fulfilled in what follows. We may state therefore:

Proposition 2.10. For $\alpha=\beta=0$ there is only one quotient (satisfying $(*)$ ) of dimension 21, say $K_{3}$, which is determined by the relation

$$
b_{2} b_{1}^{2} b_{2}+b_{1} b_{2}^{2} b_{1}+b_{1}^{2} b_{2} b_{1}+b_{1} b_{2} b_{1}^{2}+b_{1}^{2} b_{2}^{2}+b_{2}^{2} b_{1}^{2}+b_{1}+b_{2}=0 \text {. }
$$

The proof is rather calculatory and we give it in Appendix B.

We wish to study now the Markov traces on the quotient $K_{n}$ which begins with $K_{3}$, namely

$$
\begin{aligned}
& K_{n}=\left\langle 1, b_{1}, \ldots, b_{n-1}\right| b_{i+1} b_{i} b_{i+1}=b_{l} b_{l+1} b_{l} \text { for } i=1, n-1 ; b_{l} b_{j}=b_{j} b_{i} ; \\
& \qquad \begin{array}{l}
\text { for }|i-j|>1 ; b_{i}^{3}=1 \text { for all } i ; b_{l+1} b_{i}^{2} b_{l+1}=-b_{i} b_{i+1}^{2} b_{i}-b_{l}^{2} b_{l+1} b_{l} \\
\left.\quad-b_{l} b_{l+1} b_{l}^{2}-b_{i}^{2} b_{i+1}^{2}-b_{i+1}^{2} b_{i}^{2}-b_{i}-b_{l+1}\right\rangle .
\end{array}
\end{aligned}
$$

We remark first that $K_{n}$ has an obvious deformation over $\mathbf{C}^{*}$ given by

$$
\begin{aligned}
K_{n}(\gamma)= & \left\langle 1, b_{1}, \ldots, b_{n-1}\right| b_{i+1} b_{l} b_{l+1}=b_{l} b_{i+1} b_{i} \text { for } i=1, n-1 ; b_{l} b_{J}=b_{j} b_{i} ; \\
& \text { for }|i-j|>1 ; b_{l}^{3}=\gamma \text { for all } i ; b_{i+1} b_{i}^{2} b_{i+1}=-b_{l} b_{l+1}^{2} b_{l}-b_{l}^{2} b_{i+1} b_{l} \\
& \left.\quad-b_{i} b_{i+1} b_{i}^{2}-b_{l}^{2} b_{l+1}^{2}-b_{l+1}^{2} b_{i}^{2}-\gamma b_{i}-\gamma b_{l+1}\right\rangle .
\end{aligned}
$$

Remark 2.11. There is in fact exactly one solution for the system $(S)$ for general $\alpha$ and $\beta$ which is polynomial in this parameter. This was pointed out to me by P. Vogel. The reason is that $H(Q, 3)$ is a semi-simple algebra which decomposes as

$$
\mathbf{C}^{3} \oplus M_{2}^{\oplus 3} \oplus M_{3}
$$

where $M_{n}$ is the algebra of $n$ by $n$ matrices. The morphism into $\mathbf{C}^{3}$ is obtained via the abelianization map, and that into $M_{2}$ is part of the projection onto the quadratic Hecke algebra defined by a divisor of $Q$ (which is $\mathbf{C}^{2} \oplus M_{2}$ ). Therefore there is only one possibility to get a 21-dimensional quotient, by killing the factor $\mathbf{C}^{3}$. The Birman-Wenzl algebra (also called Brauer algebra in this setting) corresponds to the factor $\mathbf{C}^{2} \oplus M_{2} \oplus M_{3}$. A generator for the ideal of the quotient may be chosen as the element

$$
\begin{aligned}
\omega= & b_{1}^{2} b_{2} b_{1}^{2} b_{2}\left(b_{1} b_{2}+b_{2} b_{1}-\alpha b_{1}-\alpha b_{2}+\alpha^{2}+\beta\right)+b_{1}^{2} b_{2} b_{1}^{2}+b_{2}^{2} b_{1} b_{2}^{2} \\
& -\alpha b_{1}^{2} b_{2} b_{1}-\alpha b_{1} b_{2} b_{1}^{2}+\left(\alpha^{2}+\beta\right) b_{1} b_{2} b_{1}+b_{1} b_{2}+b_{2} b_{1}+b_{1}^{-1}+b_{2}^{-1}+\beta .
\end{aligned}
$$

The corresponding relation reduces to

$$
\begin{aligned}
& b_{2} b_{1}^{2} b_{2}+\left(\beta^{2}-\alpha\right) b_{1}^{2} b_{2}^{2} b_{1}^{2}+\left(\alpha^{2}-\alpha \beta-\beta\right)\left(b_{1}^{2} b_{2}^{2} b_{1}+b_{1} b_{2}^{2} b_{1}^{2}\right)-\alpha\left(\beta^{2}-\alpha\right) b_{1}^{2} b_{2} b_{1}^{2} \\
& +\left(1-\alpha^{3}+\alpha \beta+\alpha^{2} \beta^{2}\right)\left(b_{1}^{2} b_{2} b_{1}+b_{1} b_{2} b_{1}^{2}\right)+\left((1+\alpha \beta)^{2}-\alpha^{3}\right) b_{1} b_{2}^{2} b_{1} \\
& \quad+\left(1+2 \alpha \beta-\beta^{3}\right)\left(b_{1}^{2} b_{2}^{2}+b_{2}^{2} b_{1}^{2}\right)-\alpha\left(2+(1+\alpha \beta)^{2}-\alpha^{3}\right) b_{1} b_{2} b_{1}
\end{aligned}
$$




$$
\begin{aligned}
& +\left(\alpha \beta^{3}-2 \alpha-2 \alpha^{2} \beta\right)\left(b_{1}^{2} b_{2}+b_{2} b_{1}^{2}\right)+\left(\alpha \beta^{3}+\beta^{2}-2 \alpha^{2} \beta-2 \alpha\right)\left(b_{1} b_{2}^{2}+b_{2}^{2} b_{1}\right) \\
& +\alpha\left(2 \alpha^{2} \beta+3 \alpha-\alpha \beta^{3}-\beta^{2}\right)\left(b_{1} b_{2}+b_{2} b_{1}\right)+\left(\beta^{4}-2 \beta-3 \alpha \beta^{2}+\alpha^{2}\right)\left(b_{1}^{2}+b_{2}^{2}\right) \\
& +\left(1+3 \alpha \beta+3 \alpha^{2} \beta^{2}-\alpha \beta^{4}-\alpha^{3}\right) b_{2}+\left(1+4 \alpha \beta+3 \alpha^{2} \beta^{2}-\alpha^{3}-\alpha \beta^{4}-\beta^{3}\right) b_{1} \\
& +3 \beta^{2}-\beta^{5}-2 \alpha-3 \alpha^{2} \beta+4 \alpha \beta^{3}=0 .
\end{aligned}
$$

\section{Markov Traces on $K_{\infty}(\gamma)$}

Let us now work with the algebra $\mathbf{Z}\left[B_{\infty}\right]$ instead of $\mathbf{C}\left[B_{\infty}\right]$. Let $P_{*}$ be a quotient of $\mathbf{Z}\left[B_{\infty}\right]$. Consider $A(z, \bar{z})$ be the smallest sub-ring of $\mathbf{C}$ containing $z, \bar{z} \in \mathbf{C}^{*}$.

Definition 3.1. i) Let $R$ be a $A(z, \bar{z})$-module. The module $A F\left(P_{*}, R\right)(z, \bar{z})$ of admissible functionals on $P_{*}$ taking values in $R$ is the set of those

$$
\begin{gathered}
t \in \operatorname{Hom}_{A(z, \bar{z})}\left(P_{\infty}, R\right) \text { satisfying } \\
t\left(x b_{n} y\right)=z t(x y) \text { for } x, y \in P_{n}, \\
t\left(x b_{n}^{-1} y\right)=\bar{z} t(x y) \text { for } x, y \in P_{n} .
\end{gathered}
$$

ii) The module of Markov traces with values in $R$ is

$$
\operatorname{MT}\left(P_{*}, R\right)(z, \bar{z})=A F\left(P_{*}^{a b}, R\right)(z, \bar{z}),
$$

where $P_{k}^{a b}=P_{k} /\left[P_{k}, P_{k}\right]$ with the induced inductive system structure. Observe that $P_{k}^{a b}$ are only modules not algebras.

iii) We define the link module of $P_{*}$ with parameters $(z, \bar{z})$ as

$$
\begin{gathered}
L\left(P_{*}\right)(z, \bar{z})=P_{\infty}^{a b} /\left\langle\left\langle x b_{n}-z x, x b_{n}^{-1}-\bar{z} x ; x \in P_{n}\right\rangle\right\rangle, \\
L\left(P_{*}\right)=L\left(P_{*}\right)(1,1) .
\end{gathered}
$$

where $\langle\langle\rangle\rangle$ stands for the module spanned by the considered elements.

If $P_{*}$ is defined by homogeneous relations in each rank, then $L\left(P_{*}\right)(z, \bar{z})$, as an abelian group, is isomorphic to $L\left(P_{*}\right)$ via the map

$$
x \in P_{n} \rightarrow\left(\frac{1}{z \bar{z}}\right)^{\frac{n-1}{2}}\left(\frac{\bar{z}}{z}\right)^{\frac{e(x)}{2}} x,
$$

where $e(x)$ is the exponent sum for words. Observe also that the Markov traces descends to $L\left(P_{*}\right)(z, \bar{z})$ and we have

$$
M T\left(P_{*}, R\right)(z, \bar{z})=\operatorname{Hom}_{A(z, \bar{z})}\left(L\left(P_{*}\right)(z, \bar{z}), R\right),
$$

so the knowledge of Markov traces is enlightening when computing $L\left(P_{*}\right)$. We have natural morphisms

$$
L\left(\mathbf{Z}\left[B_{\infty}\right]\right)(z, \bar{z}) \rightarrow L\left(P_{*}\right)(z, \bar{z})
$$

and their duals

$$
\operatorname{MT}\left(P_{*}, R\right)(z, \bar{z}) \rightarrow M T\left(\mathbf{Z}\left[B_{\infty}\right]\right)(z, \bar{z}) .
$$


Let $\mathscr{L}$ be the set of isotopy classes of oriented links and $\mathbf{Z}\langle\langle\mathscr{L}\rangle\rangle$ be the free abelian group generated by $\mathscr{L}$.

Lemma 3.2. The mapping $\mathbf{Z}\langle\langle\mathscr{L}\rangle\rangle \rightarrow L\left(\mathbf{Z}\left[B_{\infty}\right]\right)$ defined by

$$
L \rightarrow \text { class of } x \text { in } L\left(\mathbf{Z}\left[B_{\infty}\right]\right) \text {, }
$$

where $x$ is some braid word representing $L$, is an isomorphism.

The proof follows from Markov's theorem in a straightforward manner.

Example 3.3. If $P_{*}=H(q, *)$ is the usual quadratic Hecke algebra then from Proposition 2.4. we derive

$$
L(H(q, *))(z, \bar{z})=\left\{\begin{array}{ll}
A(z, \bar{z}) & \text { if } z=q-1+q \bar{z} \\
0 & \text { elsewhere }
\end{array} .\right.
$$

We can state the main result of this section:

Theorem 3.4. We have

$$
L\left(K_{*}(\gamma)\right)(z, \bar{z})= \begin{cases}A(z, \bar{z}) / 6 z^{7} A(z, \bar{z}) & \text { if } z^{3}+\gamma=0, \bar{z}=-z^{2} / \gamma \\ 0 & \text { elsewhere }\end{cases}
$$

Proof. In order to get the result we need the description of Markov traces on $K_{*}(\gamma)$. First we wish to deal with the module of admissible functionals. We shall use the following type of presentation of a module:

$$
M=A\left\langle x_{1}, x_{2}, \ldots, x_{p}\left|r_{1}, r_{2}, \ldots, r_{q}\right| \mid w_{1}, w_{2}, \ldots, w_{s}\right\rangle,
$$

which has to be read as follows: $x_{1}, \ldots, x_{p}$ generates the $A$-algebra $\bar{A}$ whose defining relations are $r_{1}, \ldots, r_{q}$. Therefore $M$ is the quotient of $\bar{A}$ by the submodule spanned by the images of $w_{1}, \ldots, w_{s}$ in $\bar{A}$.

Consider now the following sets of words in the $b_{i}$ 's:

$$
\begin{aligned}
W_{1} & =\{1\}, \\
W_{n+1} & =W_{n} \cup W_{n} b_{n} Z_{n} \cup W_{n} b_{n}^{2} Z_{n}, \\
Z_{n} & =\left\{b_{n-1}^{l_{1}} b_{n-2}^{l_{2}} \ldots b_{n-p}^{i_{p}} ; i_{1}, i_{2}, \ldots, i_{p} \in\{1,2\}, p=1, n-1\right\} .
\end{aligned}
$$

First of all

Lemma 3.5. We have a surjection of $\left(K_{n}, K_{n}\right)$-bimodules

$$
K_{n} \oplus K_{n} \otimes_{K_{n-1}} K_{n} \oplus K_{n} \otimes_{K_{n-1}} K_{n} \rightarrow K_{n+1}
$$

given by

$$
x \oplus y \otimes z \oplus u \otimes v \rightarrow x+y b_{n} z+u b_{n}^{2} v .
$$

The proof follows from that of Proposition 2.6. 
As a corollary we derive that $A F\left(K_{n}, R\right)(z, \bar{z})$ is $R \otimes_{A(z, \bar{z})} M$, where $M$ has the module presentation

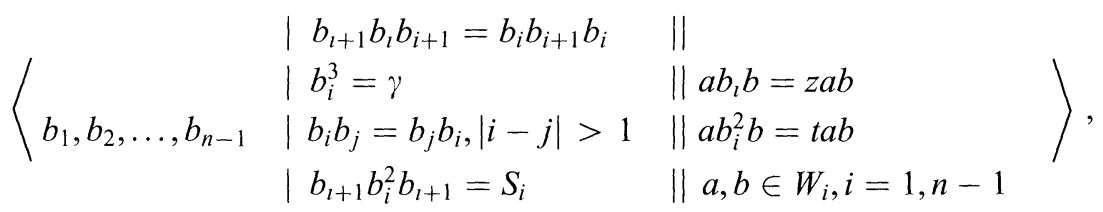

where $t=\gamma \bar{z}$,

$$
S_{i}=-\left(b_{i} b_{i+1}^{2} b_{i}+b_{i}^{2} b_{i+1}^{2}++b_{i+1}^{2} b_{l}^{2}+b_{l}^{2} b_{l} b_{l+1} b_{i}+b_{i} b_{i+1} b_{i}^{2}+\gamma b_{l}+\gamma b_{l+1}\right)
$$

and the algebra $A=A(z, \bar{z})$. We shall use this presentation for proving first

Proposition 3.6. The module of admissible functionals is

$$
A F\left(K_{*}(1), R\right)(z, \bar{z})=R / H_{0},
$$

where $H_{0}$ is the ideal $\left(12 z t^{2}+8 z^{2}-4 t, 12 z^{2} t+8 t^{2}-4 z, 10 z^{3}+10 t^{3}-2 z t-2\right) R$, and $t=1 / \bar{z}$.

We defer the rather long proof of this proposition to Sect. 5 .

We are ready now to prove our Theorem 3.4. In fact it suffices to describe the module of Markov traces taking values in $R$ for fixed parameters $(z, \bar{z})$. There is essentially only one admissible functional on $K_{\infty}(\gamma)$ from above. It suffices to check the commutativity condition

$$
t(a b)=t(b a) \text { for all } x, y .
$$

At the first stage $K_{3}(\gamma)$ we derive

$$
t\left(b_{2} b_{1}^{2} b_{2}\right)=t\left(b_{1}^{2} b_{2}^{2}\right), \quad t\left(b_{1} b_{2} b_{1}^{2} b_{2}\right)=t\left(b_{2} b_{1} b_{2} b_{1}^{2}\right)=\gamma t\left(b_{1} b_{2}\right) .
$$

But $t$ is a functional on $H(Q, 3) / I_{3}$, hence

$$
t\left(R_{0}\right)=t\left(R_{1}\right)=0 .
$$

because $I_{3}$ is spanned by $R_{0}, R_{1}, R_{2}$ (see Appendix B). These conditions imply $z^{3}+$ $\gamma=0$ and $t=-z^{2}$. So we conclude

$L\left(K_{*}(\gamma)\right)(z, \bar{z})=0$ if $z, t$ does not satisfy the previously stated conditions.

Suppose now that the parameters satisfy these conditions from now on. Therefore we see that $H_{0}=6 \gamma z^{2} R$.

We shall prove the commutativity by induction on $n$. If suffices now to check the commutativity conditions for $b \in\left\{b_{1}, \ldots, b_{n}\right\}$ and $a$ lying in a system of generators of $K_{n+1}(\gamma)$, say $W_{n+1}$. For $b=b_{i}, i<n$ it is obvious. It remains to check whenever

$$
t\left(a b_{n}\right)=t\left(b_{n} a\right) .
$$

We have three cases:

i) $a \in K_{n}(\gamma)$,

ii) $a=x b_{n} y, x, y \in K_{n}(\gamma)$,

iii) $a=x b_{n}^{2} y, x, y \in K_{n}(\gamma)$, 
which will be discussed in combination with any of the six sub-cases:

1) $x \in K_{n-1}(\gamma)$, and $y \in K_{n-1}(\gamma)$,

2) $x \in K_{n-1}(\gamma)$, and $y=u b_{n-1} v, u, v \in K_{n-1}(\gamma)$,

3) $x \in K_{n-1}(\gamma)$, and $y=u b_{n-1}^{2} v, u, v \in K_{n-1}(\gamma)$,

4) $x=r b_{n-1} s, r, s \in K_{n-1}(\gamma), y=u b_{n-1} v, u, v \in K_{n-1}(\gamma)$,

5) $x=r b_{n-1} s, r, s \in K_{n-1}(\gamma), y=u b_{n-1}^{2} v, u, v \in K_{n-1}(\gamma)$,

6) $x=r b_{n-1}^{2} s, r, s \in K_{n-1}(\gamma), y=u b_{n-1}^{2} v, u, v \in K_{n-1}(\gamma)$.

Now $\left({ }^{*}, \mathrm{i}\right),(1, \mathrm{ii})$ and $(1, \mathrm{iii})$ are trivial.

$$
\begin{aligned}
t\left(b_{n} x b_{n}^{2} u b_{n-1} v\right) & =t z t(x u v)=t\left(x b_{n} u b_{n-1} v b_{n}\right) . \\
t\left(b_{n} x b_{n}^{2} u b_{n-1} v\right) & =\gamma t\left(x u b_{n-1} v\right)=\gamma z t(x u v) \\
t\left(x b_{n}^{2} u b_{n-1} v b_{n}\right) & =t\left(x u b_{n}^{2} b_{n-1} b_{n} v\right) \\
& =t\left(x u b_{n-1} b_{n} b_{n-1}^{2} v\right)=\gamma z t(x u v) .
\end{aligned}
$$

$$
\begin{aligned}
t\left(b_{n} x b_{n} u b_{n-1}^{2} v\right) & =t^{2} t(x u v) \\
t\left(x b_{n} u b_{n-1}^{2} v b_{n}\right) & =t\left(x u b_{n} b_{n-1}^{2} b_{n} v\right) \\
& =t\left(b_{n} b_{n-1}^{2} b_{n}\right) t(x u v)=t^{2} t(x u v) .
\end{aligned}
$$

$$
\begin{aligned}
& t\left(b_{n} x b_{n}^{2} u b_{n-1}^{2} v\right)=\gamma t\left(x u b_{n-1}^{2} v\right)=\gamma t t(x u v) \\
& t\left(x b_{n}^{2} u b_{n-1}^{2} v b_{n}\right)=t\left(x u v b_{n-1} b_{n}^{2} b_{n-1}^{2} v\right)=\gamma t t(x u v) .
\end{aligned}
$$

$$
\begin{aligned}
& t\left(b_{n} r b_{n-1} s b_{n} u b_{n-1} v\right)=z t\left(r b_{n-1}^{2} s u b_{n-1} v\right), \\
& t\left(r b_{n-1} s b_{n} u b_{n-1} v b_{n}\right)=z t\left(r b_{n-1} s u b_{n-1}^{2} v\right) .
\end{aligned}
$$

Let $s u=p b_{n-2}^{\varepsilon} w$ with $p, w \in K_{n-2}(\gamma)$. If $\varepsilon=0$, it is trivial. If $\varepsilon=1$ then both terms equal $\gamma z t(r p w v)$ and if $\varepsilon=2$ again both terms equal $\gamma t t(r p w v)$ so we are done.

$$
\begin{aligned}
t\left(b_{n} r b_{n-1} s b_{n}^{2} u b_{n-1} v\right) & =t\left(r b_{n-1}^{2} b_{n} b_{n-1} s u b_{n-1} v\right) \\
& =\gamma z t\left(r s u b_{n-1} v\right)=\gamma z^{2} t(r s u v)
\end{aligned}
$$

and it is easy to check that also $t\left(r b_{n-1} s b_{n}^{2} u b_{n-1} v b_{n}\right)=\gamma z^{2} t(r s u v)$.

$$
\begin{aligned}
& t\left(b_{n} r b_{n-1} s b_{n}^{2} u b_{n-1}^{2} v\right)=\gamma z t\left(r s u b_{n-1}^{2} v\right)=\gamma z t t(r s u v), \\
& t\left(r b_{n-1} s b_{n}^{2} u b_{n-1}^{2} v b_{n}\right)=t\left(r b_{n-1} s u b_{n-1} b_{n}^{2} b_{n-1}^{2} v\right)=\gamma z t t(r s u v) .
\end{aligned}
$$


$(6$, ii $)$

$$
\begin{aligned}
t\left(b_{n} r b_{n-1}^{2} s b_{n} s b_{n} u b_{n-1}^{2} v\right)= & 3 t t\left(r b_{n-1}^{2} s u b_{n-1}^{2} v\right) \\
& -3 \gamma z t\left(r s u b_{n-1}^{2} v\right)-\gamma t\left(r b_{n-1} s u b_{n-1}^{2} v\right),
\end{aligned}
$$

and

$$
t\left(r b_{n-1}^{2} s b_{n} u b_{n-1}^{2} v b_{n}\right)=-3 t t\left(r b_{n-1}^{2} s u b_{n-1}^{2} v\right)-3 \gamma z t\left(r b_{n-1}^{2} s u v\right)-\gamma t\left(r b_{n-1}^{2} s u b_{n-1} v\right),
$$

and as in $(4$, ii $)$ we conclude that the two terms are equal.

$$
\begin{aligned}
& t\left(b_{n} r b_{n-1}^{2} s b_{n}^{2} u b_{n-1}^{2} v\right)=t\left(r b_{n-1}^{2} b_{n}^{2} b_{n-1} s u b_{n-1}^{2} v\right)=\gamma t^{2} t(r s u v) \\
& t\left(r b_{n-1}^{2} s b_{n}^{2} u b_{n-1}^{2} v b_{n}\right)=t\left(r b_{n-1}^{2} s u b_{n-1} b_{n}^{2} b_{n-1}^{2} v\right)=\gamma t^{2} t(r s u v) .
\end{aligned}
$$

$(5$, ii) The last case!

$$
\begin{aligned}
t\left(b_{n} r b_{n-1} s b_{n} u b_{n-1}^{2} v\right)= & z t\left(r b_{n-1}^{2} s u b_{n-1}^{2} v\right) \\
t\left(r b_{n-1} s b_{n} u b_{n-1}^{2} v b_{n}\right)= & -3 t t\left(r b_{n-1} s u b_{n-1}^{2} v\right)-3 \gamma z t\left(r b_{n-1} s u v\right) \\
& -\gamma t\left(r b_{n-1} s u b_{n-1}\right) .
\end{aligned}
$$

Let consider again $s u=p b_{n-2}^{\varepsilon} q$ with $p, q \in K_{n-2}(\gamma)$. If $\varepsilon=0$, it is clear.

If $\varepsilon=1$, then both terms are equal to

$$
-3 \gamma z^{2} t\left(r p b_{n-2} q v\right)-\gamma z t\left(r p b_{n-2}^{2} q v\right)-3 \gamma z t t(r p q v) .
$$

If $\varepsilon=2$, then the first term equals

$$
\gamma^{2} t\left(r p b_{n-2} q v\right)
$$

The second one turns out to be

$$
\gamma^{2} t\left(r p b_{n-2} q v\right)+6 \gamma^{2} z t(r p q v)-6 \gamma z^{2} t\left(r p b_{n-2}^{2} q v\right) .
$$

But $r$ and $v$ are arbitrary in $K_{n-1}(\gamma)$. We derive that

$$
\operatorname{MT}\left(K_{*}(\gamma), R\right)(z, \bar{z})=R /\left(6 \gamma z^{2}\right) R .
$$

When we pass to the dual we recover the result as stated in Theorem 3.4 which further implies the statement as stated in Theorem 1.3.

\section{Link Groups and Invariants}

In the last section we obtained a Markov trace

$$
t: K_{\infty}(\gamma)(z, \bar{z}) \rightarrow A(z, \bar{z}) / 6 z^{7} A(z, \bar{z})
$$

The natural way to get an invariant is to consider the function

$$
f(x)=\left(\frac{1}{z \bar{z}}\right)^{\frac{n-1}{2}}\left(\frac{\bar{z}}{z}\right)^{\frac{e(x)}{2}} t(x) .
$$


Since $\gamma=-z^{3}, \bar{z}=1 / z$ we find that $t(x)$ is an homogeneous polynomial in $z$, hence $f(x) \in \mathbf{Z}$ and does not depend upon $\gamma$ and the choice of $z$. Now the class of $f(x)$ modulo 6 is well defined and represents a Markov trace on $K_{\infty}(-1)$ with values in $\mathbf{Z} / 6 \mathbf{Z}$. By composition with the natural projection $H\left(Q_{-1}, \infty\right) \rightarrow K_{\infty}(-1)$, where $Q_{-1}=X^{3}+1$, we get an element $f \in M T\left(H\left(Q_{-1}, *\right), \mathbf{Z} / 6 \mathbf{Z}\right)(1,1)$.

Proposition 4.1. There exists a lift $F$ as a multiplicative Markov trace of $f$ in $\operatorname{MT}\left(H\left(Q_{-1}, *\right), \mathbf{Z}\right)(1,1)$ determined by

$$
F(1)=1 \text { and } F\left(b_{1}^{2} b_{2} b_{1}^{2} b_{2}\right)=-1 .
$$

Proof. Observe that $f$ is necessary as a multiplicative Markov trace on $K_{\infty}(\gamma)$, as in the quadratic case.

We need first

Lemma 4.2. If Tors $(A)$ denotes the torsion subgroup of the abelian group $A$ then

$$
2 \operatorname{Tors}\left(L\left(H\left(Q_{-1}, *\right)\right)(1,1)\right)=0 .
$$

Proof. Since $b_{l}^{3}=-1$, all the relations defining the module $L\left(H\left(Q_{-1}, *\right)\right)(1,1)$ have the following form: $w_{1}=\varepsilon w^{\prime}, \varepsilon \in\{-1,1\}$, where $w$ and $w^{\prime}$ are words in the $b_{i}$ 's. The only possibility that torsion appears will be that $w=-w$ holds, hence the torsion elements have order 2.

Assume now that $f$ is normalized by $f(1)=1$. Due to the form of the relations $R_{0}, R_{1}, R_{2}$ we obtain $f(x)=\varepsilon$ (modulo 6 ), $\varepsilon \in\{-1,1\}$ if $x$ is a word. Then the previous lemma enables us to get a lift.

$$
F \in M T\left(H\left(Q_{-1}, *\right), \mathbf{Z}\right)(1,1),
$$

whose reduction modulo 6 is $f$. Remark that $f$ is a Markov trace so its values on $e_{1}, e_{2}, \ldots, e_{23}$ are uniquely determined from $f(1)=1, z=1, t=-1$. The only freedom degree in the definition of $F$ (on $H\left(Q_{-1}, 3\right)$ ) is the choice of

$$
F\left(b_{1}^{2} b_{2} b_{1}^{2} b_{2}\right)=-7+6 k \text {. }
$$

We remark now that $f$, restricted to words, takes only the values 1 and -1 (modulo 6). Therefore the application $F: B_{n} \rightarrow \mathbf{Z}$ defined as

$$
F(x)=\left\{\begin{array}{ll}
1 & \text { if } f(x)=1 \text { (modulo 6) } \\
-1 & \text { otherwise }
\end{array},\right.
$$

extends naturally to a Markov trace on $H\left(Q_{-1}, *\right)$ taking integer values, so we can choose $k=1$ above. We denote by the same letter the link invariant which is associated to $F$.

We think that a Markov trace on $H(Q, *)$ exists for any choice of $k$, but it is hard to believe that it is algorithmically computable.

Hopefully we may compute algorithmically $F(x)$ since its reduction modulo 6 (which is $F(x)$ itself!) is $f$ so we can use the algorithm described in the previous section. This ends the proof of the proposition.

Proposition 4.3. The invariant $F$ is not a Vassiliev invariant of finite degree.

Proof. We shall consider $K$ the classical torus knot of type $(1,12 k)$ and set $K^{(12 k)}$ for the singular knot having all crossings identified. We remark that $F\left(b_{1}^{j}\right)=\sigma(j)$, 
where $\sigma$ has period 6 and $\sigma(0)=\sigma(1)=\sigma(5)=1, \sigma(2)=\sigma(3)=\sigma(4)=-1$. So $\sigma(j)=\sigma(-j)$. Let $F$ denote also the extension of $F$ to singular knots. According to [BXS93] we may write

$$
F\left(K^{(12 k)}\right)=\sum_{p=0}^{12 k} C_{12 k}^{p}(-1)^{p} \sigma(2 p-12 k)=\sum_{p=0}^{12 k} C_{12 k}^{p}(-1)^{p} \sigma(2 p),
$$

where $C_{n}^{k}$ states for the number of subsets of $k$ elements of a cardinal $n$ set. Let $\zeta=\exp \left(\frac{2 \pi i}{3}\right)$ and

$$
a_{j}=\sum_{p=0 ; p=j(3)}^{12 k}(-1)^{p} C_{12 k}^{p}, j \in\{0,1,2\}
$$

Then

$$
F\left(K^{(12 k)}\right)=a_{0}-a_{1}-a_{2},
$$

so from some elementary combinatorics we derive

$$
F\left(K^{(12 k)}\right)=\frac{4}{3} 27^{2 k}
$$

which proves our claim.

This ends the proof of our Main Theorem 1.2.

We don't know however if $F$ is not the limit of a sequence of Vassiliev invariants. On the other hand $F$ generates a whole sequence of Vassiliev type invariants, as follows. Let $S B_{n}$ be the monoid of singular braids (see [BXS93, Bir93]) with generators $g_{i}, g_{i}^{-1}, s_{i}, 1 \leqq i<n$ and relations

$$
\begin{aligned}
& {\left[g_{i}, g_{j}\right]=\left[s_{i}, g_{J}\right]=\left[s_{l}, s_{j}\right]=0 \quad \text { if }|i-j|>1, \quad\left[g_{l}, s_{l}\right]=0,} \\
& g_{i+1} g_{i} g_{i+1}=g_{i} g_{i+1} g_{l}, \quad s_{l+1} g_{i} g_{i+1}=g_{l} g_{i+1} s_{l} .
\end{aligned}
$$

Let $\mathrm{ZSB}_{n}$ be the monoid algebra of the singular braid monoid. The (3-order) Vassiliev algebra $W_{n}$ is defined as the quotient of $\mathbf{Z} S B_{n} \otimes \mathbf{Z}[\varepsilon]$ by the ideal generated by the following elements:

$$
g_{i}^{2}+g_{i}^{-1}=\varepsilon s_{i} .
$$

If $\mathbf{Z}(\varepsilon)$ denotes the algebra of Laurent polynomials in $\varepsilon$, then it is clear that the natural map $i: \mathbf{Z}\left[B_{n}\right] \rightarrow W_{n}$ produces an isomorphism

$$
\mathbf{Z}\left[B_{n}\right] \otimes \mathbf{Z}(\varepsilon) \rightarrow W_{n} \otimes \mathbf{Z}[\varepsilon] \mathbf{Z}(\varepsilon) .
$$

Now any link invariant $I$ will extend to singular links admitting transverse double points by means of the following resolution rule for the singularities:

$$
\varepsilon I\left(L_{x}\right)=I\left(L_{++}\right)+I\left(L_{-}\right),
$$

where $L_{x}, L_{++}, L_{-}$denotes the link diagrams with an intersection, two left-handed crossings and one right-handed crossing respectively, all the rest of the diagrams being the same (see picture 20). A link invariant is of degree $d$ if it vanishes on all singular links with $d+1$ or more self intersections. A Markov trace on $W_{\infty}$ taking 
values in the $A(z, \bar{z})[\varepsilon]$-module $E$ is a map $t \in \operatorname{Hom}_{A(z, \bar{z})[\varepsilon]}\left(W_{\infty}, E\right)$ satisfying

$$
\begin{aligned}
& t(x y)=t(y x) \quad \text { for all } x, y, \\
& t\left(x g_{n}\right)=z t(x), \quad t\left(x g_{n}^{-1}\right)=\bar{z} t(x) \quad \text { if } x \in W_{n} .
\end{aligned}
$$

We say that a Markov trace on $W_{\infty}$ is homogeneous of degree $d$ if for every $x \in Z\left[B_{\infty}\right], t(i(x))$ is a homogeneous polynomial in $\varepsilon$ of degree $d$.

Proposition 4.4. There is a one-to-one correspondence between the Markov traces

$$
t \in M T\left(\mathbf{Z}\left[B_{\infty}\right]\right), A(z, \bar{z})(z, \bar{z})
$$

whose associated invariant $I$ is of degree $d$ and Markov traces $\tau: W_{\infty} \rightarrow A(z, \bar{z})[\varepsilon]$ that are homogeneous of degree $d$, given by

$$
\tau(i(x))=\varepsilon^{d} t(x) .
$$

Proof. Suppose that $I$ is of degree $d$. The formula from above defines a Markov trace on $W_{\infty}$ with values in $A(z, \bar{z})(\varepsilon)$. Let $x \in W_{n}$ which is a product of $l$ elements of the form $s_{j}$ and arbitrarily many of the form $g_{j}$. Then

$$
\tau(x)=\varepsilon^{d-l}(z \bar{z})^{\frac{n-1}{2}}\left(\frac{z}{z}\right)^{\frac{e(x)}{2}} I(\hat{x}),
$$

where $\hat{x}$ denotes the closure of the singular braid $x$. Remark that $\hat{x}$ has exactly $l$ self-intersections. So if $l>d, I(\hat{x})$ vanishes hence $\tau$ takes values in $A(z, \bar{z})[\varepsilon]$.

Conversely assume $\tau$ is given and satisfies the hypothesis. Then $t$ is a Markov trace. Let $L$ be a link with $l$ self-intersections. Then $L$ is isotopic to some $\hat{x}$, where $x \in S B_{n}$ may be written

$$
x=w_{1} s_{i_{1}} w_{2} s_{i_{2}} w_{3} \ldots w_{l} s_{l} w_{l+1}
$$

with $w_{l}$ words in the $g_{l}$ 's. Set

$$
x^{\prime}=w_{1}\left(g_{i_{1}}^{2}+g_{i_{1}}^{-1}\right) w_{2}\left(g_{i_{2}}^{2}+g_{i_{2}}^{-1}\right) w_{3} \ldots w_{l}\left(g_{i_{l}}^{2}+g_{i_{1}}^{-1}\right) w_{l+1} .
$$

Therefore

$$
\varepsilon^{d} t(x)=\tau(i(x))=\varepsilon^{l} \tau\left(x^{\prime}\right) .
$$

Assume that $l>d$. Since $\tau\left(x^{\prime}\right) \in \mathbf{C}[\varepsilon]$ and $t(x) \in \mathbf{C}$, we derive that $t(x)=0$, hence $I(L)=0$.

We can obtain therefore a necessary and sufficient condition that an invariant be the limit of $\left(3^{\text {rd }}\right.$ order $)$ Vassiliev invariants of finite degree.

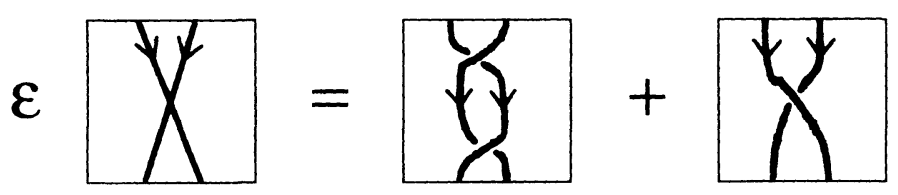

Fig. 1. The resolution of a self-intersection singularity. 
Proposition 4.5. The invariant I associated to the Markov trace $t$ is the limit of a Vassiliev invariant of finite degree iff there exists a Markov trace

$$
\tau: W_{\infty} \rightarrow A(z, \bar{z})[\varepsilon]
$$

which makes the following diagram commutative:

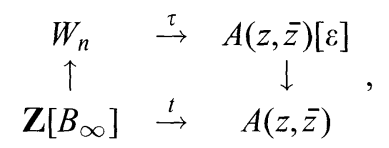

where the morphism $A(z, \bar{z})[\varepsilon] \rightarrow A(z, \bar{z})$ is given by $\varepsilon \rightarrow 1$.

Proof. If $t=\sum_{d} t_{d}$, where $t_{d}$ are of degree $d$ then take $\tau=\sum_{d} \varepsilon^{d} \tilde{t}_{d}$, where $\tilde{t}$ is the natural extension of $t_{d}$ to $W_{\infty}$ and conversely. The rest of the proof is similar to that of the previous proposition.

Now we observe that in the quadratic Hecke algebra the following relation:

$$
b_{j}^{2}+b_{j}^{-1}=\left(q^{2}-q+1\right) q^{-1}\left(b_{i}-1\right)
$$

holds. This implies that the Markov trace with parameter $z$, say $t_{q, z}$ fulfills the following condition:

$$
t_{q, z}\left(w_{1}\left(g_{l_{1}}^{2}+g_{l_{1}}^{-1}\right) w_{2}\left(g_{l_{2}}^{2}+g_{i_{2}}^{-1}\right) w_{3} \ldots w_{l}\left(g_{i_{l}}^{2}+g_{i_{l}}^{-1}\right) w_{l+1}\right)=(q-\zeta)^{l} c
$$

where $\zeta^{3}=-1$ and $c \in \mathbf{C}$.

Corollary 4.6. If $H(q, z)(*)$ is the Homfly polynomial then

$$
H(\zeta \exp (q), z)(L)=\sum_{j=1}^{\infty} w_{l}(L, z) q^{i},
$$

where $w_{l}(*, z)$ are Vassiliev invariants of degree $i$.

The proof follows from the previous proposition, when we take $\varepsilon=q-\zeta$.

In particular nontrivial Vassiliev invariants of every degree exist. In the same manner we can prove that all quantum invariants of Turaev are obtained as the limit of $3^{\text {rd }}$ order Vassiliev invariants.

We remark that all constructions we made could actually be performed by replacing $x^{2}+x^{-1}$ by any polynomial $Q$. If $Q$ is quadratic then only classical Vassiliev invariants are obtained. Consider $Q=x+1$ and call the induced invariants $1^{\text {st }}$ order Vassiliev invariants. The previous results may be restated word-by-word also for this case. Let $V(i)^{d}$ denote the space of Vassiliev invariants of order $i$ (coming from some polynomial $Q$ of degree $i$ ) and degree $d$ and $\bar{V}(i)^{\infty}$ the space of invariants which are limits of finite degree invariants. We remark that the quantum invariants are already contained in $\bar{V}(1)^{\infty}$. Consider the case of the Homfly polynomial. We make first a change of variable in the Homfly polynomial by setting $h=\frac{1}{q}$. Then in the quadratic Hecke algebra $H(q, \infty)$ we have

$$
b_{i}+1=h(h-1)^{-1}\left(b_{i}^{2}+1\right) \text {. }
$$

Therefore the Homfly polynomial $H(h, z)(k)$, when developing in a series the factor $\bar{z}^{-1}$ which normalizes the trace, has the coefficients $1^{\text {st }}$ order Vassiliev invariants. It 
is simple to check that

$$
V(2)^{d} \cap V(3)^{\dagger} \subset V(1)^{d+f}
$$

which implies that

$$
\bar{V}(2)^{\infty} \cap \bar{V}(3)^{\infty}=\bar{V}(1)^{\infty} .
$$

Whenever these three spaces are distinct we don't know. However the space of $1^{\text {st }}$ order invariants seems to be more treatable from the algebraic viewpoint.

\section{Graphical Reduction of Obstructions}

This section will be concerned with the proof of Proposition 3.6. Firstly from Lemma 3.5 we derive that an admissible functional, if one ever exists, is unique up to the choice of $t(1) \in R$. Look now at the algebra $K_{n}(\gamma)$. We wish to use the following transforms on the words:

$$
\begin{aligned}
b_{i+1} b_{i} b_{l+1} & \rightarrow b_{l} b_{l+1} b_{l}, \\
b_{i}^{3} & \rightarrow \gamma, \\
b_{l+1} b_{l}^{2} b_{l+1} & \rightarrow S_{l},
\end{aligned}
$$

and only in this direction, in order to reduce the degree of $b_{n-1}$ as much as possible. According to Lemma 3.5 every word is equivalent to a sum of words of type $\sum_{l} x_{l} b_{n-1}^{i_{l}} y_{l}$. Unfortunately we are forced to use the relations

$$
b_{1} b_{1} \Leftrightarrow b_{1} b_{1} \text { for }|i-j|>1 \text {, }
$$

in both directions. Assume this is the process we shall carry out. So we obtain finally a sum $\sum_{l} x_{l} b_{n-1}^{i_{i}} y_{l}$ with $x_{l}, y_{l} \in K_{n-1}(\gamma)$. Of course this "normal form" for the word we started with is not unique since we may perform again permutations of its letters in each term. But if any two such normal forms would be equilvalent under eventual permutations of its letters (of $b_{1} b$, with $|i-j|>1$ always!) we should have an almost canonical description of the basis of $K_{n}(\gamma)$. Indeed the last assumption is equivalent to say that the surjection of Lemma 3.5 is actually an isomorphism. However this is not the case. We can at least obtain the obstructions to the uniqueness of this almost canonical form. We return now to the module of admissible functionals. The last group of relations enables us to make a further reduction, namely

$$
\begin{aligned}
& a b_{n-1} b \longrightarrow z a b, \\
& a b_{n-1} b \longrightarrow t a b .
\end{aligned}
$$

This way we may reduce finally a word to a sum of words lying in $K_{n-2}(\gamma)$. Assume that we are using a recurrence on $n$. Then each element of $K_{n-2}\left(\gamma^{\prime}\right)$ may be uniquely reduced to an element of $R$ (the value of the functional on the element). So it suffices to check the obstructions directly on the values in order to obtain that the functional is well-defined. That is what we shall formalize now.

Definition 5.1. A semi-oriented graph is a graph having two types of edges: oriented edges and unoriented ones. A path $v_{1} v_{2} \ldots v_{n}$ is a semi-oriented path if for all $j$ or $v_{1} \rightarrow v_{1+1}$ or else $v_{j} v_{1+1}$ is unoriented. If all edges are unoriented we say that its endpoints are unoriented equivalents. 
The Pentagon Condition 5.2. The pentagon condition for semi-oriented graphs can be stated as follows:

(PC) If $v_{2} \rightarrow v_{1}, v_{2} v_{3} \ldots v_{n-1}$ is an unoriented path, $v_{n-1} \rightarrow v_{n}$ then there exists semi-oriented paths $v_{1} x_{1} x_{2} \ldots x_{m} e$ and $v_{n} y_{1} y_{2} \ldots y_{p}$ e having the same endpoint (see Fig. 2).

We say now that $x \leqq y$ if there exists an semi-oriented path from $y$ to $x$ in $\Gamma$. Of course $\leqq$ is not always a partial order relation. It is necessary and sufficient that no closed semi-oriented loops exist in $\Gamma$. However we can say that $x$ is minimal if $y \leqq x$ implies that $y$ is unoriented equivalent to $x$.

Pentagon Lemma 5.3. Suppose that the (PC) holds. If a connected component $C$ of the graph $\Gamma$ has a minimal element $m_{C}$ then it is unique up to unoriented equivalence.

Proof of lemma. Consider two minimal elements $x$ and $y$ which lie in $C$. Then there exists some path $x x_{0} x_{1} \ldots x_{n} y$ joining them. Since $x$ is minimal the closest oriented edge (if one ever exists) is in-going, and the same is true for $y$. If this path is not unoriented and again from minimality there are at least two oriented edges. Therefore open pentagon configurations (i.e. those configurations where (PC) applies) exist. We apply then (PC) iteratively whenever such configurations exist or have appeared. When this process stops we find two semi-oriented $x z_{1} z_{2} \ldots z_{p} e$ and $y u_{1} u_{2} \ldots u_{s} e$ having the same endpoint $e$. So $e \leqq x$ and $x \leqq y$. Again from minimality these paths must be unoriented so $x$ and $y$ are unoriented equivalent (see Fig. 3).

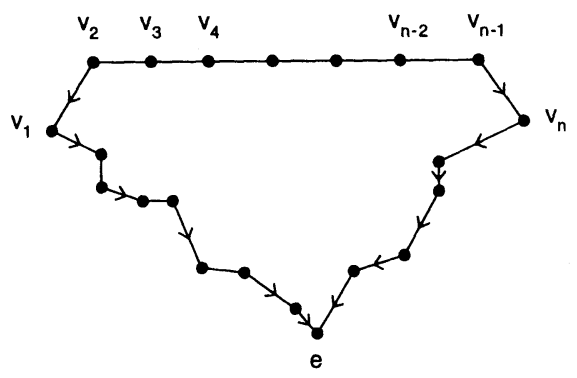

Fig. 2. The Pentagon Condition.

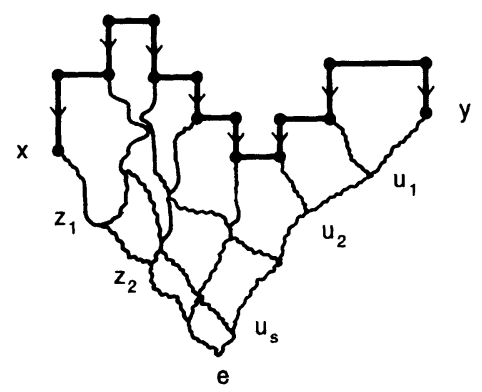

Fig. 3. Proof of Pentagon Lemma. 
We remark that a priori we can say nothing about the existence of such minimal elements. If $\leqq$ would be a partial order with descending chain condition then the existence of minimal elements is standard. However in the case which we shall work out even if $\leqq$ is not a partial order the existence of minimal elements can be established.

Suppose now we have a more complicated structure: a sequence of disjoint graphs $\Gamma_{n}$. In every $\Gamma_{n}$ there exists a distinguished subset of vertices $V_{n}^{0}$ which are minimal elements in their connected components. Suppose that each connected component admits at least one minimal element. Each such vertex from $V_{n}^{0}$ has exactly one outgoing edge going to a vertex of $\Gamma_{n-1}$. We color these new edges in red. Set $\Gamma_{n}^{*}$ for the union of all $\Gamma_{j}, j \leqq n$ and with the red edges added in each rank $j$.

Definition 5.4. We say that $\Gamma_{n}^{*}$ is coherent if any connected component of $\Gamma_{n}$ has an unique minimal element (with respect to $\Gamma_{n}^{*}$ ) in $\Gamma_{0}$ up to unoriented equivalence.

We shall state now the colored version of the Pentagon Lemma for this type of graph. We introduce:

Colored Pentagon Condition 5.5. (CPC) If $v_{1} v_{2} \ldots v_{n}$ is an open pentagon configuration in $\Gamma_{n}$, then there exists bicolored semi-oriented paths (in $\Gamma_{n}^{*}$ ) from $v_{1}$ and $v_{2}$ having the same endpoint.

In addition if $x y$ is an unoriented edge in $\Gamma_{n}$ with $x, y \in V_{n}^{0}$ then there exist semi-oriented paths in $\Gamma_{n}^{*}$ starting with red edges and having the same endpoint (see Fig. 4).

Colored Pentagon Lemma 5.6. Suppose that $\Gamma_{n-1}^{*}$ is coherent and the (CPC) condition is fulfilled. Then $\Gamma_{n}^{*}$ is coherent.

The proof is similar to that of the Pentagon Lemma.

We are ready to define now our graph $\Gamma_{n}$. Its vertices are the elements of the group algebra of the free monoid $F_{n}$ in $(n-1)$ letters $\left\{b_{1}, b_{2}, \ldots, b_{n-1}\right\}$. Two vertices $v=\sum_{i} \alpha_{l} x_{i}$ and $w=\sum_{l} \beta_{i} y_{i}, \alpha_{i}, \beta_{i} \in A(z, \bar{z})$ are related by an oriented edge if exactly one monomial of $v$ is changed following one of the rules:

(C0) (j) $A b_{j}^{3} B \rightarrow A B$

(C1)(j) $A b_{j+1} b_{j} b_{j+1} B \rightarrow A b_{j} b_{j+1} b_{j} B$,

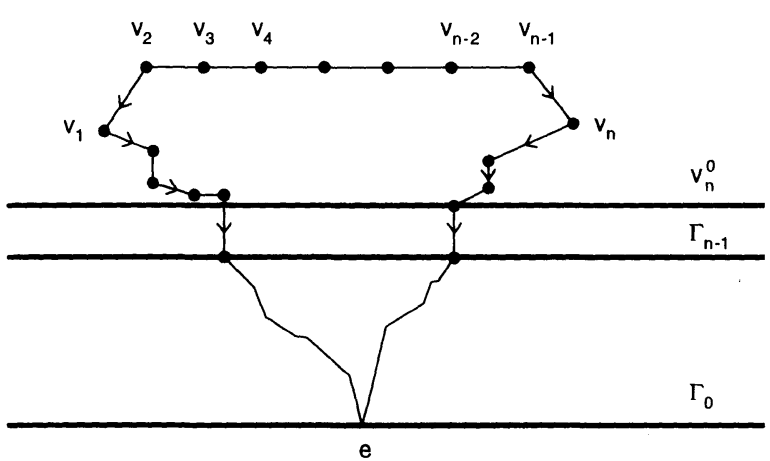

Fig. 4. The colored pentagon condition. 
(C2) (j) $A b_{j+1} b_{j}^{2} b_{j+1} B \rightarrow A S_{j} B$

(C12)(j) $A b_{j+1} b_{j}^{2} b_{j+1}^{2} B \rightarrow A b_{j}^{2} b_{j+1}^{2} b_{j} B$,

(C21)(j) $A b_{j+1}^{2} b_{j}^{2} b_{j+1} B \rightarrow A b_{j} b_{j+1}^{2} b_{j}^{2} B$.

Also an unoriented edge between $v$ and $w$ correspond to a change in a monomial of $v$ of type

$$
\left(P_{l j}\right) A b_{l} b_{j} B \rightarrow A b_{j} b_{i} B \quad \text { whenever }|i-j|>1 .
$$

We remark that the use of $(\mathrm{C} 12)$ and $(\mathrm{C} 21)$ is somewhat ambiguous since we may always use ( $\mathrm{C} 2)$ for a sub-word. Their role is to break in some sense the closed oriented loops in $\Gamma_{n}$. In fact consider $V_{n}^{0}$ to be the set of vertices corresponding to elements of the free abelian $A=A(z, \bar{z})$-module generated by $W_{n}$.

Lemma 5.7. Each connected component of $\Gamma_{n}$ has a minimal element in $V_{n}^{0}$, not necessarily unique.

Proof of lemma. We prove our claim by induction on $n$. For $n=1$ it is trivial. Say now $w$ is a word in the $b_{i}$ 's having only positive exponents. If its degree in $b_{n-1}$ is zero or one we apply the induction hypothesis and we are done. If the degree is 2 and only one $b_{n-1}^{2}$ we are already in a position to apply the induction hypothesis. Also we may suppose that no exponents greater than 2 occur by using (C0) several times. If the degree is 2 then $w=x b_{n-1} y b_{n-1} z$ with $x, y, z \in F_{n-1}$. The induction applied to $y$ implies that $w \geqq x b_{n-1} a b_{n-2}^{\varepsilon} b z$ with $a, b \in F_{n-2}$. Then several transforms of type $\left(P_{n-1 j}\right)$ and $(C \varepsilon)$ will do the job. Consider now that the degree is strictly greater than 2 . So we have a sub-word of type

$$
b_{n-1}^{\alpha} x b_{n-1}^{\beta} \quad \text { with } 3 \leqq \alpha+\beta \leqq 4
$$

or else one of the type $b_{n-1} x b_{n-1} y b_{n-1}$. The second case reduces to the first one as above. Next assume that $x \geqq a b_{n-2}^{\varepsilon} b . a, b \in F_{n-2}$. Several applications of $\left(P_{n-1 j}\right)$ leads us to consider the word $b_{n-1}^{\alpha} b_{n-2}^{\varepsilon} b_{n-1}^{\beta}$. If $\varepsilon=1$ we apply two times (C1) and we are done. Otherwise we shall apply $(\mathrm{C} \alpha \beta)$ if $\alpha \neq \beta$ or both $(\mathrm{C} 12)$ and $(\mathrm{C} 21)$ if $\alpha=\beta=2$. This proves that every vertex descends to $V_{n}^{0}$. But these vertices have not outgoing edges in $\Gamma_{n}$ as can be easily seen. When we use the unoriented edges some new vertices have to be added, which are not in $V_{n}^{0}$. But it is easy to see that these also do not have outgoing edges. Since any vertex has a semi-oriented path ending in $V_{n}^{0}$ we are done.

We remark that the moves $(\mathrm{C} 12)$ and $(\mathrm{C} 21)$ are really necessary for the conclusion of the above lemma to remain valid. In fact from $b_{2} b_{1}^{2} b_{2}^{2}$ only (C2) may be applied. We obtain a factor $b_{2}^{2} b_{1}^{2} b_{2}$. If we continue, at each stage we shall find one of these two monomials. When all reductions are used at the second stage we recover $b_{2} b_{1}^{2} b_{2}^{2}$ so we have a closed oriented loop in the graph. Its connected component should have a minimal element without the use of $(\mathrm{C} 12)$ or (C21).

We are able now to define the bicolored graph $\Gamma_{n}^{*}(z, t)$. The red edges are added as follows: Each minimal vertex $v=\sum_{i} \alpha_{l} x_{l} b_{n}^{\varepsilon_{l}} y_{l}$, is related to $w=\sum_{l} \alpha_{i} z_{i} x_{i} y_{i}$ which this time is a vertex of $\Gamma_{n-1}$, where we met $z_{0}=1, z_{1}=z, z_{2}=t$. Since $b_{j}^{3}=\gamma$ we have $t=\gamma \bar{z}$ and we prefer working with $(z, t)$ instead with the couple of parameters $(z, \bar{z})$. Finally we define $\Gamma_{0}(H)$ as the graph having the vertices corresponding to the module $R$. Two vertices are connected by an unoriented edge iff the corresponding elements lie in the same coset of $R / H, H$ being a certain 
submodule of $R$. Let also $H_{0}=\left(12 z t^{2}+8 z^{2}-4 t, 12 z^{2} t+8 t^{2}-4 z, 10 z^{3}+10 t^{3}-\right.$ $2 z t-2) R$ be a fixed submodule of $R$. We specify the choice of $H$ in the tower bicolored graph by denoting it $\Gamma_{n}^{*}(H)$, even if only the first stage depends upon $H$. With these notations we can state:

Proposition 5.8. The bicolored graph $\Gamma_{\infty}^{*}(H)$ is coherent if and only if $H_{0} \subset H$.

Before we proceed we remark that this result has as an immediate corollary Proposition 3.6. Indeed for $H=H_{0}$ we know how to associate to each element of $K_{n}(\gamma)$ an element of $R$. The only problem which we can encounter is that moving on different descending paths we should obtain different elements. But the previous proposition states the uniqueness of the endpoint viewed as an element of $R / H_{0}$. So our claims follows.

Proof. We shall prove the coherence of each $\Gamma_{n}^{*}(H)$ by recurrence on $n$. For $n=1,2$ this may be easily established without any condition on $H$. We wish to make use the Colored Pentagon Lemma. For instance we shall look only at the Pentagon Condition in $\Gamma_{n}$. For those configurations that we cannot prove the (PC) directly we shall check that the (CPC) (which is weaker since it regards all of $\Gamma_{n}^{*}(H)$ ) is still verified. Of course this implies that we apply the Colored Pentagon Lemma.

Definition 5.9. An open pentagon configuration (abbrev. o.p.c.) is a sequence of vertices $\left[w_{0}, w_{1}, \ldots, w_{n}\right]$ such that $w_{1} \rightarrow w_{0}, w_{1}, \ldots, w_{n-1}$ are unoriented equivalent and $w_{n-1} \rightarrow w_{n}$. We say that this o.p.c. is irreducible if none of the vertices $w_{1}, w_{2}, \ldots, w_{n-1}$ has an outgoing edge.

Reduction Lemma 5.10. i) In order to verify (PC) it suffices to restrict to irreducible configurations.

ii) It suffices to verify (PC) only for monomials from $F_{n}$.

iii) Suppose $w_{j}^{\prime}=A w_{j} B$, for $j=0, n$ (so $A, B$ are not touched by any transform), in the o.p.c.. If (PC) holds for $\left[w_{0}, w_{1}, \ldots, w_{n}\right]$ it also holds for $\left[w_{0}^{\prime}, w_{1}^{\prime}, \ldots, w_{n}^{\prime}\right]$.

iv) Suppose that (PC) holds for $\left[w_{0}, w_{1}, \ldots, w_{n}\right]$ and for $\left[y_{0}, y_{1}, \ldots, y_{m}\right]$. Then for all $A, B, C$ the (PC) is valid also for

$$
\begin{gathered}
{\left[A w_{0} B y_{1} C, A w_{1} B y_{1} C, \ldots, A w_{n-1} B y_{1} C, A w_{n-1} B y_{2} C,\right.} \\
\left.A w_{n-1} B y_{3} C, \ldots, A w_{n-1} B y_{m-1} C, A w_{n-1} B y_{m} C\right] .
\end{gathered}
$$

In fact when we fix the endpoints of the o.p.c. we can mix the unoriented edges of each subjacent o.p.c. in any order we want. Let $\left(i_{k}, j_{k}\right) \in\{0,1, \ldots, n\} \times$ $\{0,1, \ldots, m\}, k=1, p$ such that $i_{0}=0<i_{1} \leqq i_{2} \leqq \cdots \leqq i_{p}, j_{p}=m>j_{p-1} \geqq$ $\cdots \geqq 0$, and $i_{k+1}-i_{k}+j_{k+1}-j_{k}=1$ for all $k$. Then the o.p.c.

fulfills the (PC).

$$
\left[A w_{i_{0}} B y_{j_{0}} C, A w_{l_{1}} B y_{J_{1}} C, \ldots, A w_{i_{p}} B y_{j_{p}} C\right]
$$

Proof. i) We may always decompose a configuration into irreducible ones and iterate the construction.

ii) The reduction transforms on different monomials commute with each other, so we are done.

iii) Obvious.

iv) The reductions of $x_{n-1}$ and $y_{1}$ commute again with each other.

Thus the top line of an o.p.c. corresponds to a word $w_{1}$ and a sequence of permutations of its letters giving in order $w_{2}, w_{3}, \ldots, w_{n-1}$. We may suppose that $w=w_{1}$ 
has no proper sub-words $w_{1}^{\prime}$ which fulfill the following two conditions:

i) Say $w=A w^{\prime} B$. Then each of the considered permutations acts on the letters of $A$, of $B$ or $w^{\prime}$. So there is an equivalent of $w^{\prime}$ say $w^{\prime \prime}$.

ii) The reduction transforms performed at $w_{1}$ and $w_{2}$ acts actually on $w^{\prime}$ and $w^{\prime \prime}$.

In other words only those letters may be not permuted which enter in a block which is reduced. Also it follows that of $n=2$, so the top line is trivial and exactly two outgoing edges are incident in $w_{1}$ or else the corners $w_{1}$ and $w_{n-1}$ have each exactly one outgoing edge, except for the case when a move (Cij) may be applied. When such an edge ( $\mathrm{Cij}$ ) exists then of course also $(\mathrm{C} 2)$ exists so there are two outgoing edges. We shall choose always the edge ( $\mathrm{Cij}$ ) if one ever exists and we shall say that the corresponding word has unique reduction. If ever in our o.p.c. the outgoing edge from $w$ is an (C2), even if a (Cij) may be performed, then we know that a semi-oriented loop exists permitting to come back in $w$. So the (PC) is trivially satisfied.

Now the top line is determined by the sequence of transpositions of the letters of $w$. Let $l$ be the length of $w$. Otherwise this is the same as giving a permutation $\sigma \in$ $S_{l}$ with a prescribed decomposition into transpositions. Set $T_{l}$ for the transposition which interchanges the letters on the positions $l$ and $l+1$. Notice that for a fixed $w$ not all $\sigma$ are suitable. In fact only a subset of the group of permutations, which we call permitted may work. Say $P(w)$ is the set of permitted permutations. If $e_{w}:\{1,2, \ldots, l\} \rightarrow\{1,2, \ldots, n-1\}$ is the evaluation map

$$
e_{w}(j)=\text { index of the letter lying in position } j \text { on } w,
$$

then $T_{j} \sigma$ is permitted (where $\sigma \in P(w)$ ) iff

$$
\left|e_{\sigma(w)}(j)-e_{\sigma(w)}(j+1)\right|>1 .
$$

Say that two permitted permutations $\sigma$ and $\sigma^{\prime}$ are equivalent if for the o.p.c. corresponding to $\sigma$ and $\sigma^{\prime}$ the (PC) is valid or not for both at the same time.

Lemma 5.11. i) Suppose that $\sigma_{1} T_{j} T_{i} \sigma_{2} \in P(w),|i-j|>1$. Then $\sigma_{1} T_{l} T_{j} \sigma_{2} \in P(w)$ and these two permutations are equivalent.

ii) Suppose that $\sigma_{1} T_{i+1} T_{i} T_{i+1} \sigma_{2} \in P(w)$. Then $\sigma_{1} T_{i} T_{1+1} T_{1} \sigma_{2} \in P(w)$ and these two permutations are equivalent. The converse is still true.

iii) If $\sigma_{1} T_{i}^{2} \sigma_{2} \in P(w)$ then $\sigma_{1} \sigma_{2}$ is permitted and equivalent to previous one.

Proof. The existence in the first case is equivalent to

$$
\left|e_{\sigma_{2}(w)}(j)-e_{\sigma_{2}(w)}(j+1)\right|>1
$$

and

$$
\left|e_{\sigma(w)}(i)-e_{\sigma(w)}(i+1)\right|>1,
$$

so is symmetric. In the second case also it is equivalent to

$$
\left|e_{\sigma_{2}(w)}\left(j+\varepsilon_{1}\right)-e_{\sigma_{2}(w)}\left(j+\varepsilon_{2}\right)\right|>1 \text { for all } \varepsilon_{j} \in\{0,1,2\} .
$$

so it is again symmetric. The equivalence is trivial.

We shall use a graphical representation for the decomposition of $\sigma$ into transpositions similar to the braid pictures (see Picture 5), where we specify on the top and 


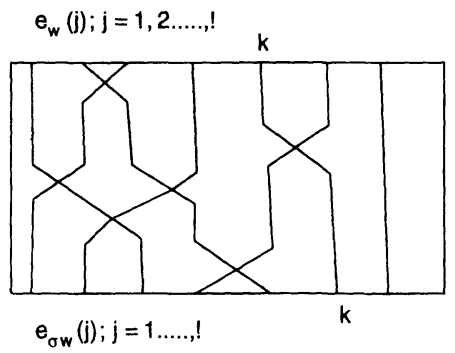

Fig. 5. The complete diagram associated to an o.p.c.

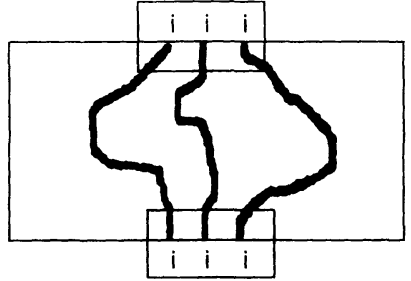

a

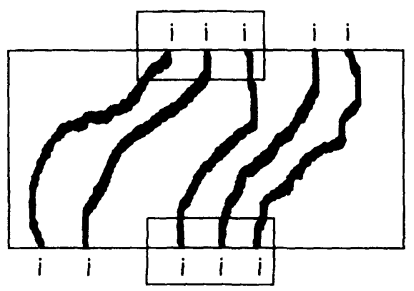

c

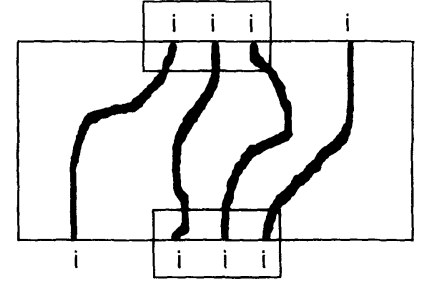

b

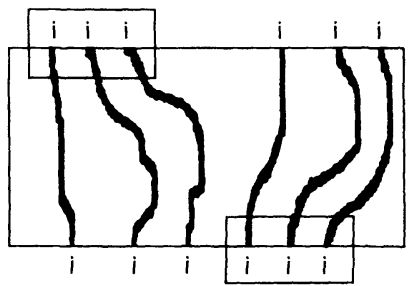

d

Fig. 6. The essential trajectories for $(\mathrm{CO})(\mathrm{i})-(\mathrm{C} 0)(\mathrm{i})$.

bottom lines of the diagram the values of the evaluation maps. This picture encodes all information about the o.p.c. because the two words $w$ and $\sigma(w)$ have unique reduction. Firstly we are interested only in drawing the trajectories of the six (to ten) elements which enter in the two blocks which reduces. Suppose for instance that the two reduction moves are two $(\mathrm{C} 0)$. So $w=x i i i y$ and $\sigma(w)=x^{\prime} j j j y^{\prime}$. Say that $i=j$. The trajectories of the $i$ 's may be disjointed since the transposition acting on the couple $i i$ is trivial in fact. So the possible trajectories fit into 4 cases which may be seen in Picture 6.a,b, c, d.

Suppose now we have two trajectories of $i$ and $j \neq i$ which intersect. First of all we derive that $|i-j|>1$. Orient all the arcs from the top to the bottom.

Lemma 5.12. i) Suppose that the arcs labeled $i$ and $j$ have algebraic intersection number 0 . Then we can replace the diagram by an equivalent one where the arcs are disjoint.

ii) Suppose that the arcs labeled $i$ and $j$ have algebraic intersection number 1. Then we can replace the diagram by an equivalent one where the arcs have exactly one intersection point. 


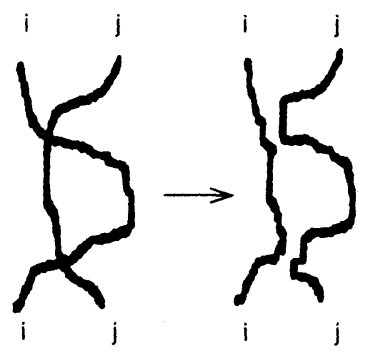

Fig. 7. Disjointing trajectories.

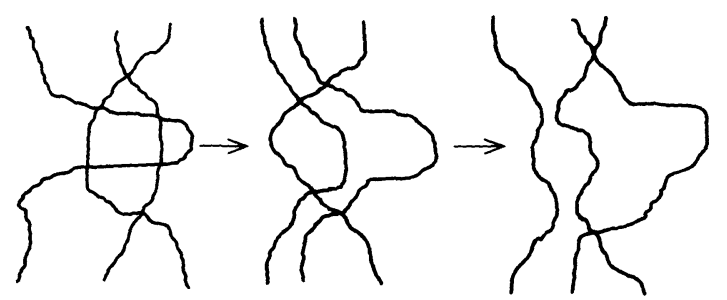

Fig. 8. Non-minimal biangle's procedure.

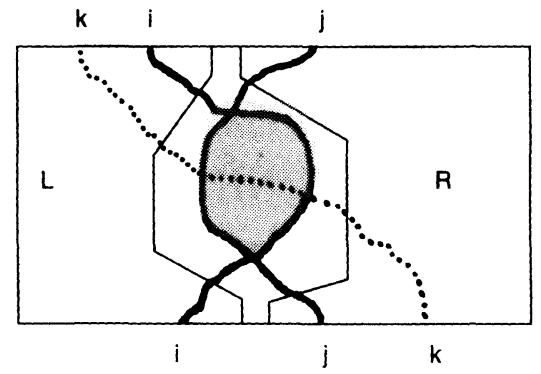

Fig. 9. The regions $R$ and $L$.

Proof. We consider the diagram is that from Fig. 7.

We can assume that the biangle in the middle is minimal, hence it does not contain any other biangle. In fact we can apply repeatedly the disjointedness procedure only for minimal biangles. Such a biangle has two walls: one coming from $i$ and the other from $j$. From minimality no other arc crosses twice the small wall (see Picture 8).

Let consider the region $L$ and $R$ such that: the set of arcs labeled by something not commuting with $j$ is contained in $L$, and those labeled by some $k$ not commuting with $i$ are contained in $R$. Then the situation is that from Picture 9.

Thus all arcs which cross the biangle are labeled by some $k$ which commutes with both $i$ and $j$. The same commutation transforms may be performed whenever we make the arcs $i$ and $j$ disjoint.

A similar reasoning permits to say that the diagrams from Picture 10 are equivalent. When the triangle in the middle is not touched by any arc then it is a simple 
consequence of Lemma 3.12 ii). If it is minimal, any arc which crosses it is labeled by something which commutes with $j$.

We remark now the similitude of Pictures 7 and 10 with the Reidemester's moves on link diagrams. So we can actually isotopy our arcs leaving the endpoints fixed and keeping the tangent (in a $C^{1}$-approximation of arcs) away from the horizontal.

Now we can continue our discussion on the trajectories of $i$ 's and $j$ 's. If $|i-j|=$ 1 the trajectories are disjoint so there are as in Picture 11.

If $i$ and $j$ commutes there are essentially sixteen diagrams (up to an isotopy) which can be seen in Picture 12.

In order to represent graphically the possible diagrams for the $(\mathrm{C} 1),(\mathrm{C} 2),(\mathrm{C} 12)$, (C21) moves we shall picture the trajectories of a couple of neighbor points having the same label as a single thicker trajectory. This may be done since every arc crossing the dashed region (see Fig. 13) between the trajectories of the two $i$ 's has a label commuting with $i$. In addition the trajectories of $i$ and $i+1$ are disjoint.

Suppose we are in the case (C1)(i)-(C0)(j). For $j \neq i-1, i, i+1, i+2$ the sixteen diagrams from above appear appropriately labeled. For $j=i-1, i, i+2$ some diagrams are not realized because the arcs labeled by $i-1$ and $i$ do not intersect, so several cases have to be left. For $j=i+1$ another diagram has to be considered, that from Fig. 14.

The same situation we encounter when we describe the possible trajectories for the couple of reduction transforms $(\mathrm{C} 2)-(\mathrm{C} 0),(\mathrm{C} 12)-(\mathrm{C} 0),(\mathrm{C} 21)-(\mathrm{C} 0)$. A simple analysis shows that in the remaining cases the only new diagrams are those from Fig. 15.
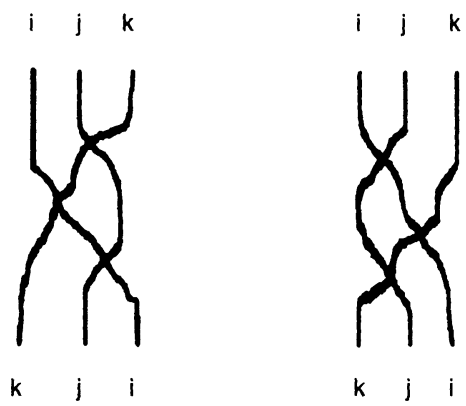

Fig. 10. Equivalent diagrams.

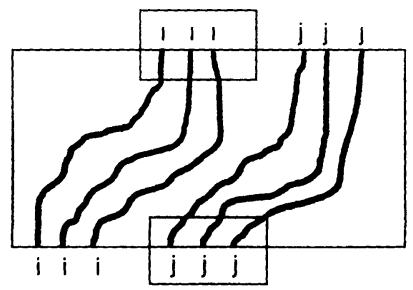

Fig. 11. The diagram for $(\mathrm{C} 0)(\mathrm{i})-(\mathrm{C} 0)(\mathrm{j})$ when $|i-j|>1$. 

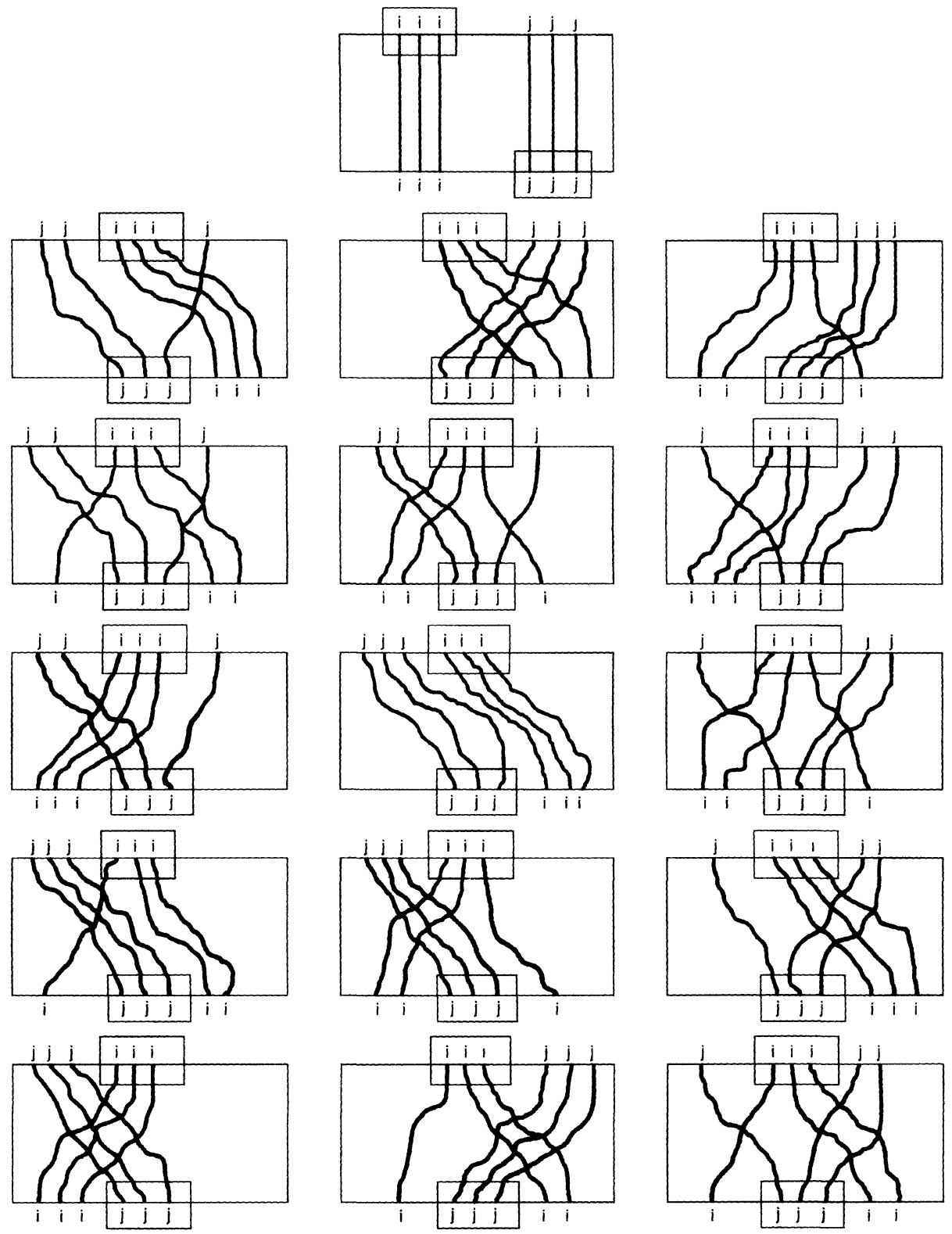

Fig. 12. The 16 diagrams for $(\mathrm{C} 0)(\mathrm{i})-(\mathrm{C} 0)(\mathrm{j})$ in the commuting case.

The other ones are obtained from the previous twelve using suitable labeling, and taking into account the constraints of disjointedness imposed by the labels. We say now that a diagram is interactive if there is some marked arc relating the top and bottom blocks where the reduction transforms act. Our task will be to eliminate the non-interactive diagrams where the (PC) trivially holds.

Lemma 5.13. The usual (PC) is valid in $\Gamma_{n}$ for non-interactive diagrams. 


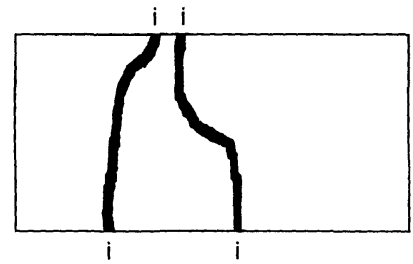

Fig. 13. The graphical representation of the dashed region.

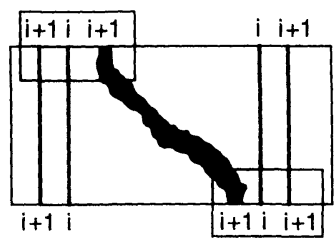

Fig. 14. The new diagram for $(\mathrm{C} 1)(\mathrm{i})-(\mathrm{C} 0)(\mathrm{i}+1)$.
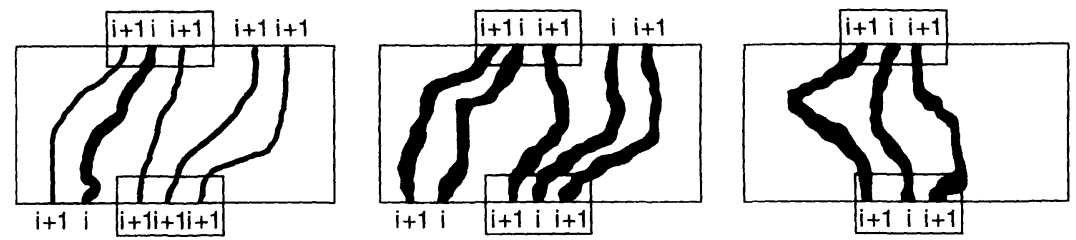

Fig. 15. The new diagrams for $(\mathrm{Cx})(\mathrm{i})-(\mathrm{Cy})(\mathrm{i}) x, y \neq 0$.

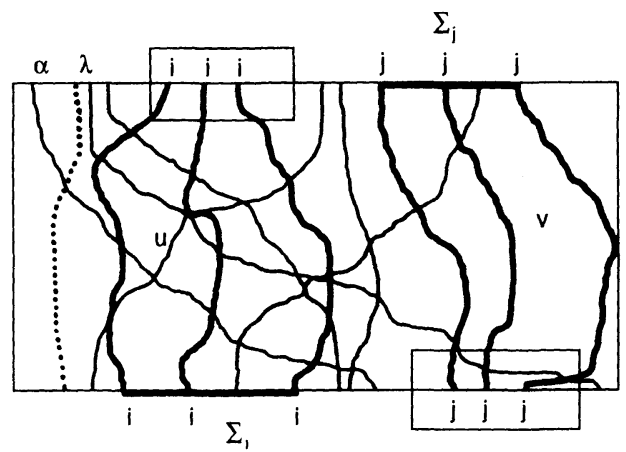

Fig. 16. The whole picture of a non-interactive diagram without crossings.

Proof. We consider first the case where no crossings of the essential arcs exist. The typical case is that from Picture 10. We draw now all trajectories as in Fig. 16. We have the dashed regions $U$ and $V$ which are bounded by the $i$ 's arcs and respectively $j$ 's arcs.

Everything crossing the regions $U$ and $V$ commutes with $i$ and $j$ respectively. We claim first that $U$ and $V$ are tangent to the end lines from left and right respectively. If not there exists some arc labeled $\lambda$ lying to the left of $U$. Assume that this arc is the first from the left having this property. In particular $\lambda$ commutes with every label $\alpha$ which stands to the left of $\lambda$. Thus we may perform these 
commutation transforms at any moment, to get $\lambda$ on the first position. Since $\lambda$ does not cross $U$ we may leave it on the first position replacing the o.p.c. by an equivalent one. Thus the new configuration corresponds to a word which is not minimal with respect to the reduction procedure (see the reduction lemma and the subsequent comments).

Let now $\Sigma_{l}$ be the convex hull of the three points labeled $i$ coming from essential arcs and lying on the bottom line. Similarly set $\Sigma_{j}$ for the convex hull of the $j$ 's on the top line. Every arc which arrive on $\Sigma_{l}$ must cross $U$, hence is labeled by some $k$ commuting with $i$. We can move these endpoints using the commutation rules from the left or the right according to the following principle: if the startpoint of the arc labeled $k$ is in the left of the block of $i$ 's on the top line, then we move to the left. Otherwise we move to the right. The only problem which we can have is in the following case: the start-point of some $k$ is in the left of the arc labeled $l$, both arrive on $\Sigma_{l}$, but this time the endpoint of $l$ is in the left of $k$. A topological argument shows that these two arcs cross each other. Therefore $k$ and $l$ are commuting and we can perform our transforms as it was said (see Fig. 17).

Finally we shall recover a diagram which this time has crossings but is equivalent to the standard one of Picture 18.

Suppose now that the reduction transforms $A i i i B \rightarrow A B$ and $C j j j D \rightarrow C D$ are also performed. We may use the simplification transforms (commutations which are

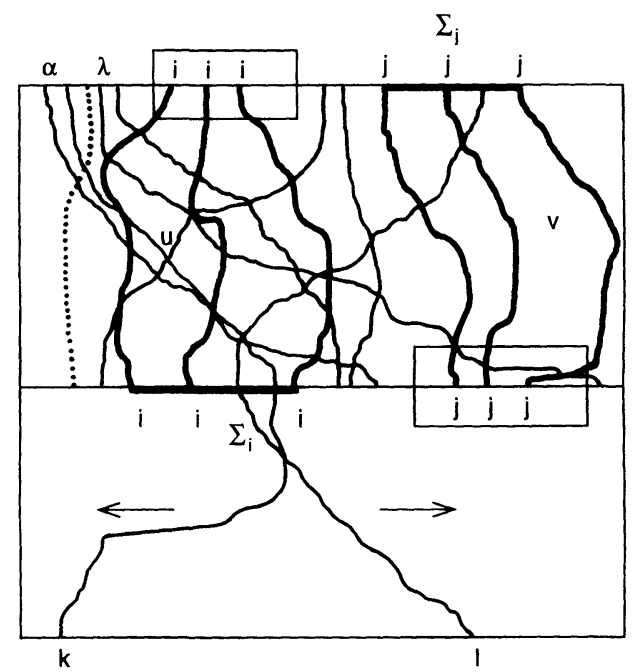

Fig. 17. The simplification of a non-interactive diagram without crossings of essential arcs.

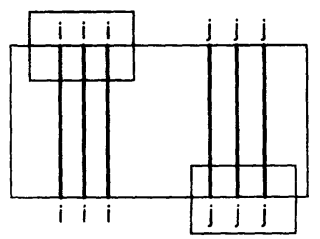

Fig. 18. The standard non-interactive diagram. 
still valid even if the $i$ or the $j$ are collapsed) above for each word: to $A B$ in the part of $j$ 's and to $C D$ in the part of $i$ 's. Due to the particular form of the standard diagram we shall get (see Picture 16) the words $U j j j V$ and $U^{\prime} i i i V^{\prime}$ respectively, with $U V=U^{\prime} V^{\prime}$. So again the use of a reduction transform will get the same word. Thus the (PC) is satisfied for these configurations. It is almost the same reasoning for the other non-interactive diagrams without crossings.

It remains the case when crossings of essential arcs appear. But the commutation transforms may also be performed in such a way that the starting points of $j$ 's on the top line will be all on the same part with respect to the iii block. In other words we make $\Sigma_{j}$ and the block iii disjoints. The same is true for the bottom line. The worst case is again when iii is in the left of $\Sigma_{j}$ on the top line and down the situation is reversed. But again $i$ and $j$ commute with everything which starts or arrives on the convex hulls of $i i i \cup \Sigma_{j}$ and $j j j \cup \Sigma_{i}$. So we can rearrange them to obtain the same order in the top and bottom lines. This ends the proof of the lemma.

So it remains to look at the interactive configuration. The same reasoning as in the above permits us to restrict to the normal forms drawn in Fig. 19.a-f. Some of the trajectories may be thick trajectories.

The cases $a, b, c, d, f$ are trivially verified because only the consistency of relations defining $K_{3}(\gamma)$ is involved.

Let us do a sub-case of $\mathrm{d}$, corresponding to $(\mathrm{C} \varepsilon)-\mathrm{C}(0)$ : The monomial has the form $\omega=b_{l+1} b_{i}^{\varepsilon} b_{i+1} x b_{l+1}^{2}$ which is unoriented equivalent to $w^{\prime}=b_{l+1} b_{l} \varepsilon b_{l+1}^{3}$. Here $x$ commutes with $b_{i+1}$, so we may suppose it lies in $F_{l}$. Therefore $x \rightarrow$ $x_{0} b_{l-1}^{j_{1}} b_{l-2}^{j_{2}} \ldots b_{l-p}^{j_{p}}$, with $x_{0} \in F_{i-1}$. So again we can restrict to the case $x_{0}=1$. Now $w$ is reduced to $S b_{i-1}^{j_{1}} b_{i-2}^{j_{2}} \ldots b_{l-p}^{j_{p}} b_{l+1}^{2}$. This is equivalent to $S b_{l+1}^{2} b_{l-1}^{j_{1}} b_{l-2}^{j_{2}} \ldots b_{i-p}^{j_{p}}$. Since $S b_{i+1}^{2}$ and $b_{l+1} b_{i}^{\varepsilon}$ may be related by a semi-oriented path to a common endpoint (here we use the induction hypothesis for $n=2$ ) we are done. All other cases are similar.

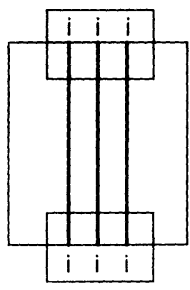

a

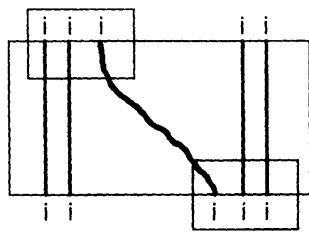

b

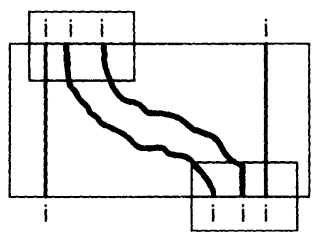

C

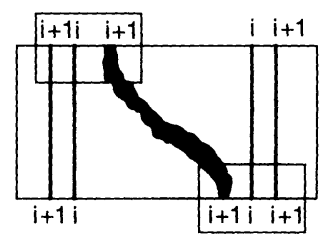

$\mathbf{e}$

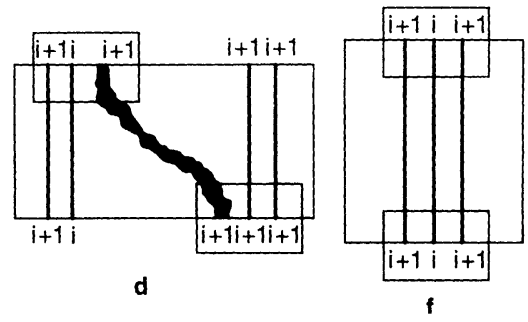

Fig. 19. The normal forms of interactive configurations. 
In the case e the situation is different. Using the commutation rules, as above we must preserve the term $b_{i-1}^{j_{1}}$. So we must check the configurations

$$
w=x b_{i+1}^{\alpha} b_{i}^{\varepsilon} b_{i+1}^{\beta} b_{i-1}^{\mu} b_{i}^{\delta} b_{i+1}^{\gamma} b_{i-2}^{j_{2}} \ldots b_{i-p}^{j p},
$$

where $x \in F_{i-1}$ and $\alpha, \beta, \gamma$ equal 1 if $\varepsilon=\delta=1$. It is here where we cannot prove that the (PC) holds. In fact it does not hold since the surjection of Proposition 3.5 has a nontrivial kernel in rank $n=3$. Fortunately we may prove that the (CPC) condition is still verified.

Lemma 5.14. For the above family of o.p.c. the (CPC) is satisfied in $\Gamma_{n}^{*}(H), n \geqq 3$ iff $H_{0} \subset H$.

Proof. We claim first that it suffices to restrict to $x=1, p=1$. We observe that any admissible functional $t$ on $K_{\infty}(\gamma)$ satisfies

$$
t(x u v)=t(u) t(x v) \text { for } x, v \in H(Q, m) \text { and } u \in\left\langle 1, b_{m}, b_{m+1}, \ldots, b_{m+k}\right\rangle .
$$

If $k=0$ this is nothing but the multiplicative property for Markov traces. If $k>0$ then in the process which computes the value of the functional we replace $u$ by $\alpha b_{m}^{\varepsilon}$, where $t(u)=\alpha t\left(b_{m}^{\varepsilon}\right)$. Another one step of the reduction and we found $t(x u v)=$ $\alpha t\left(b_{m}^{\varepsilon}\right) t(x v)$.

But the (CPC) is equivalent to the existence of the admissible functional. This proves our claim which says that in fact we can consider $i=2$. Consider first $\alpha=\beta=\gamma=1$. We have to check the o.p.c. corresponding to

$$
b_{3} b_{2}^{\varepsilon} b_{3} b_{1}^{\mu} b_{2}^{\delta} b_{3} \text {. }
$$

In order to shorten the computations we observe that for symmetric words the (CPC) is satisfied. For a word $w=w_{1}, \ldots, w_{l}$ its symmetric is $w^{*}=w_{l} w_{l-1}, \ldots, w_{1}$. This has a simple proof by induction. In fact we perform in both situations the same reduction transforms (the words have unique reduction) which leads us to two sums of minimal elements $\sum_{i} x_{l}$ and $\sum_{l} x_{l}^{*}$. Now we apply the induction hypothesis and we are done. Roughly speaking

$$
t(x)=t\left(x^{*}\right)
$$

for any admissible functional $t$. So it suffices to check the case when

$$
\varepsilon=1 \quad \text { and } \quad \delta=2 \text {. }
$$

1) Say $\mu=1$. Then

$$
\begin{gathered}
w=b_{3} b_{2} b_{3} b_{1} b_{2}^{2} b_{3} \rightarrow b_{2} b_{3} b_{1}^{2} b_{2} b_{1} b_{3} \sim b_{2} b_{1}^{2} b_{3} b_{2} b_{1} \rightarrow z b_{2} b_{1}^{2} b_{2}^{2} b_{1} \rightarrow z b_{1}^{2} b_{2}^{2} b_{1}^{2} \rightarrow \gamma t z^{2} \\
w^{\prime}=b_{3} b_{2} b_{1} b_{3} b_{2}^{2} b_{3} \rightarrow-b_{3} b_{2} b_{1} b_{2} b_{3}^{2} b_{2}-b_{3} b_{2} b_{1} b_{2}^{2} b_{3} b_{2}-b_{3} b_{2} b_{1} b_{2} b_{3} b_{2}^{2} \\
-b_{3} b_{2} b_{1} b_{2}^{2} b_{3}^{2}-b_{3} b_{2} b_{1} b_{3}^{2} b_{2}^{2}-\gamma b_{3} b_{2} b_{1} b_{3}-\gamma b_{3} b_{2} b_{1} b_{2}
\end{gathered}
$$

Now

$$
\begin{gathered}
b_{3} b_{2} b_{1} b_{2} b_{3}^{2} b_{2} \rightarrow b_{3} b_{1} b_{2} b_{1} b_{3}^{2} b_{2} \sim b_{1} b_{3} b_{2} b_{3}^{2} b_{1} b_{2} \\
\rightarrow b_{1} b_{2}^{2} b_{3} b_{2} b_{1} b_{2} \rightarrow z b_{1} b_{2}^{3} b_{1} b_{2} \rightarrow \gamma t z^{2} \\
b_{3} b_{2} b_{1} b_{2}^{2} b_{3} b_{2} \rightarrow b_{3} b_{1}^{2} b_{2} b_{1} b_{3} b_{2} \rightarrow b_{1}^{2} b_{2} b_{3} b_{2} b_{1} b_{2} \rightarrow z b_{1}^{2} b_{2}^{2} b_{1} b_{2} \rightarrow z b_{1}^{3} b_{2} b_{1}^{2} \rightarrow \gamma t z^{2}
\end{gathered}
$$




$$
\begin{gathered}
b_{3} b_{2} b_{1} b_{2} b_{3} b_{2}^{2} \rightarrow b_{3} b_{1} b_{2} b_{1} b_{3} b_{2}^{2} \rightarrow b_{1} b_{2} b_{3} b_{2} b_{1} b_{2}^{2} \rightarrow z b_{1} b_{2}^{2} b_{1} b_{2}^{2} \\
\rightarrow z b_{1}^{2} b_{2} b_{1}^{2} b_{2} \rightarrow-6 \gamma z^{2} t-\gamma^{2} z \\
b_{3} b_{2} b_{1} b_{2}^{2} b_{3}^{2} \rightarrow b_{3} b_{1}^{2} b_{2} b_{1} b_{3}^{2} \rightarrow b_{1}^{2} b_{3} b_{2} b_{3}^{2} b_{1} \rightarrow b_{1}^{2} b_{2}^{2} b_{3} b_{2} b_{1} \rightarrow \gamma^{2} z \\
b_{3} b_{2} b_{1} b_{3}^{2} b_{2}^{2} \rightarrow b_{3} b_{2} b_{3}^{2} b_{1} b_{2}^{2} \rightarrow b_{2}^{2} b_{3} b_{2} b_{1} b_{2}^{2} \rightarrow \gamma t^{2} z \\
b_{3} b_{2} b_{1} b_{3} \rightarrow b_{2} b_{3} b_{2} b_{1} \rightarrow \gamma z^{2} t \\
b_{3} b_{2} b_{1} b_{2} \rightarrow z b_{1} b_{2} b_{1} \rightarrow \gamma t z^{2} .
\end{gathered}
$$

We conclude

$w^{\prime}=b_{3} b_{2} b_{1} b_{3} b_{2}^{2} b_{3} \rightarrow \gamma z^{2} t$ thus the (CPC) is verified.

2) Consider now $\mu=2$. We shall write all details up to the use of red edges where we arrive at $\Gamma_{2}^{*}$ and the computation becomes canonical:

$$
\begin{gathered}
w=b_{3} b_{2} b_{3} b_{1}^{2} b_{2}^{2} b_{3} \sim w^{\prime}=b_{3} b_{2} b_{1}^{2} b_{3} b_{2}^{2} b_{3} \\
w \rightarrow-b_{2} b_{1}^{2} b_{2} b_{3}^{3} b_{2} b_{1}-b_{2} b_{1}^{2} b_{2}^{2} b_{3} b_{2} b_{1}-b_{2} b_{1}^{2} b_{2} b_{3} b_{2}^{2} b_{1}-b_{2} b_{1}^{2} b_{2}^{2} b_{3}^{3} b_{1}-b_{2} b_{1}^{2} b_{3}^{2} b_{2}^{2} b_{1} \\
-\gamma b_{2} b_{1}^{2} b_{2} b_{1}-\gamma b_{2} b_{1}^{2} b_{3} b_{1} \rightarrow-b_{1}^{2} b_{2}^{2} b_{1} b_{3} b_{2} b_{1}-b_{1}^{2} b_{2}^{2} b_{1} b_{3}^{2} b_{1}-b_{2} b_{1}^{2} b_{3}^{2} b_{2}^{2} b_{1} \\
-\gamma b_{2} b_{1}^{2} b_{3} b_{1}+b_{1} b_{2}^{2} b_{1} b_{3}^{2} b_{2} b_{1}+b_{1}^{2} b_{2} b_{1} b_{3}^{2} b_{2} b_{1}+b_{1} b_{2} b_{1}^{2} b_{3}^{2} b_{2} b_{1}+b_{1}^{2} b_{2}^{2} b_{3}^{2} b_{2} b_{1} \\
+b_{2}^{2} b_{1}^{2} b_{3}^{2} b_{2} b_{1}+\gamma b_{2} b_{3}^{2} b_{2} b_{1}+\gamma b_{1} b_{3}^{2} b_{2} b_{1}+b_{1} b_{2}^{2} b_{1} b_{3} b_{2}^{2} b_{1}+b_{1}^{2} b_{2} b_{1} b_{3} b_{2}^{2} b_{1} \\
+b_{1} b_{2} b_{1}^{2} b_{3} b_{2}^{2} b_{1}+b_{1}^{2} b_{2}^{2} b_{3} b_{2}^{2} b_{1}+b_{2}^{2} b_{1}^{2} b_{3} b_{2}^{2} b_{1}+\gamma b_{2} b_{3} b_{2}^{2} b_{1}+\gamma b_{1} b_{3} b_{2}^{2} b_{1} \\
-\gamma b_{2} b_{1}^{2} b_{2} b_{1} \rightarrow-4 \gamma^{2} t-3 \gamma t^{2} z
\end{gathered}
$$

On the other hand

$$
\begin{aligned}
w^{\prime} \rightarrow & -b_{3} b_{2} b_{1}^{2} b_{2} b_{3}^{2} b_{2}-b_{3} b_{2} b_{1}^{2} b_{2}^{2} b_{3} b_{2}-b_{3} b_{2} b_{1}^{2} b_{2} b_{3} b_{2}^{2}-b_{3} b_{2} b_{1}^{2} b_{2}^{2} b_{3}^{2} \\
& -b_{3} b_{2} b_{1}^{2} b_{3}^{2} b_{2}^{2}-\gamma b_{3} b_{2} b_{1}^{2} b_{3}-\gamma b_{3} b_{2} b_{1}^{2} b_{2} .
\end{aligned}
$$

The last four terms are easy to compute:

$$
\begin{aligned}
b_{3} b_{2} b_{1}^{2} b_{2} & \rightarrow-\left(3 \gamma t^{2} z+4 \gamma^{2} z^{2}\right), \\
b_{3} b_{2} b_{1}^{2} b_{3} & \rightarrow t^{2} z, \\
b_{3} b_{2} b_{1}^{2} b_{3}^{2} b_{2}^{2} & \rightarrow b_{2}^{2} b_{3} b_{2} b_{1}^{2} b_{2}^{2} \rightarrow \gamma t^{2} z, \\
b_{3} b_{2} b_{1}^{2} b_{2} b_{3}^{2} & \rightarrow b_{1} b_{2}^{2} b_{3}^{2} b_{2} b_{1} \rightarrow \gamma^{2} t .
\end{aligned}
$$

For the other ones the computation becomes more complicated. We shall write it this time as an array where the reduction of each term is specified. 


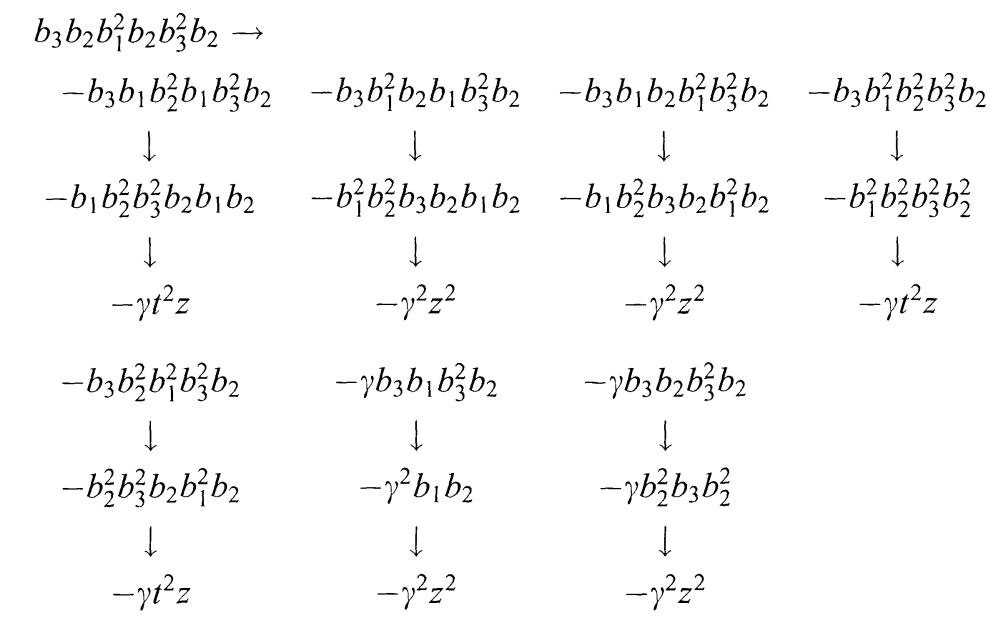

so we obtain

$$
b_{3} b_{2} b_{1}^{2} b_{2} b_{3}^{2} b_{2} \rightarrow-\left(3 \gamma t^{2} z+4 \gamma^{2} z^{2}\right) .
$$

Also

$$
\begin{aligned}
b_{3} b_{2} b_{1}^{2} b_{2}^{2} b_{3} b_{2} \rightarrow & b_{1}^{2} b_{3} b_{2}^{2} b_{3} b_{1} b_{2} \\
\rightarrow & -b_{1}^{2} b_{2} b_{3}^{2} b_{2} b_{1} b_{2}-b_{1}^{2} b_{2}^{2} b_{3} b_{2} b_{1} b_{2}-b_{1}^{2} b_{2} b_{3} b_{2}^{2} b_{1} b_{2} \\
& -b_{1}^{2} b_{2}^{2} b_{3}^{2} b_{1} b_{2}-b_{1}^{2} b_{3}^{2} b_{2}^{2} b_{1} b_{2}-\gamma b_{1}^{2} b_{3} b_{1} b_{2}-\gamma b_{1}^{2} b_{2} b_{1} b_{2} \\
\rightarrow & -\left(3 \gamma t^{2} z+4 \gamma^{2} z^{2}\right) .
\end{aligned}
$$

The last term is given by

$$
\begin{array}{cccc}
-b_{3} b_{2} b_{1}^{2} b_{2} b_{3} b_{2}^{2} \rightarrow & & \\
b_{3} b_{1} b_{2}^{2} b_{1} b_{3} b_{2}^{2} & +b_{3} b_{1}^{2} b_{2} b_{1} b_{3} b_{2}^{2} & +b_{3} b_{1} b_{2} b_{1}^{2} b_{3} b_{2}^{2} & +b_{3} b_{1}^{2} b_{2}^{2} b_{3} b_{2}^{2} \\
& \downarrow & \downarrow & \downarrow \\
& b_{1}^{2} b_{2} b_{3} b_{2} b_{1} b_{2}^{2} & b_{1} b_{2} b_{3} b_{2} b_{1}^{2} b_{2}^{2} & -\left(6 \gamma t^{2} z+\gamma^{2} t\right) \\
& \downarrow & \downarrow & \\
+b_{3} b_{2}^{2} b_{1}^{2} b_{3} b_{2}^{2} & -\left(3 \gamma t^{2} z+4 \gamma^{2} z^{2}\right) & \gamma t^{2} z & \\
\downarrow & +\gamma b_{3} b_{2} b_{3} b_{2}^{2} & +\gamma b_{3} b_{1} b_{3} b_{2}^{2} & \\
-\left(6 \gamma t^{2} z+\gamma^{2} t\right) & \downarrow & \downarrow & \downarrow
\end{array}
$$

and

$$
\begin{array}{cccc}
b_{3} b_{1} b_{2}^{2} b_{1} b_{3} b_{2}^{2} \rightarrow & & & \\
-b_{1} b_{2} b_{3}^{2} b_{2} b_{1} b_{2}^{2} & -b_{1} b_{2}^{2} b_{3} b_{2} b_{1} b_{2}^{2} & -b_{1} b_{2} b_{3} b_{2}^{2} b_{1} b_{2}^{2} & -b_{1} b_{2}^{2} b_{3}^{2} b_{1} b_{2}^{2} \\
\downarrow & \downarrow & \downarrow & \downarrow \\
6 \gamma t^{2} z+\gamma^{2} t & -\gamma t^{2} z & -\gamma t^{2} z & 6 \gamma z t^{2}+\gamma^{2} t \\
-b_{1} b_{3}^{2} b_{2}^{2} b_{1} b_{2}^{2} & -\gamma b_{1} b_{2} b_{1} b_{2}^{2} & -\gamma b_{1} b_{3} b_{1} b_{2}^{2} & \\
\downarrow & \downarrow & \downarrow & \\
6 \gamma z t^{2}+\gamma^{2} t & -\gamma^{2} z^{2} & -\gamma t^{2} z &
\end{array}
$$


hence

$$
b_{3} b_{1} b_{2}^{2} b_{1} b_{3} b_{2}^{2} \rightarrow 15 \gamma t^{2} z+3 \gamma^{2} t-\gamma^{2} z^{2} .
$$

We introduce in the previous expression and obtain for the last term

$$
b_{3} b_{2} b_{1}^{2} b_{2} b_{3} b_{2}^{2} \rightarrow \gamma^{2} t+2 \gamma t^{2} z-4 \gamma^{2} z^{2},
$$

so the final result is

$$
w^{\prime} \rightarrow 9 \gamma t^{2} z+8 \gamma^{2} z^{2}
$$

This way we get the first obstruction $12 z t^{2}+8 z^{2}-4 t=0$ (modulo $H_{0}$ ). The other configurations we must check are with $\alpha, \beta, \gamma$ greater than 1 . A computer search was used to verify that the hand computations in the remaining cases are correct and give the other two obstructions. Thus the (CPC) holds for the considered o.p.c. We remark that the second part of the (CPC) is trivially satisfied when using an induction. This ends the proof of Lemma 3.15.

It follows now we may apply the Colored Pentagon Lemma to get the coherence of $\Gamma_{n}^{*}\left(H_{0}\right)$ so the proof of Proposition 3.10 which we observed implies the result of Proposition 3.6.

\section{A. Appendix: The Module $H(Q, 3)$}

This part of the paper is joint work with Barbu Berceanu.

We shall get in this section the complete proof of Proposition 2.1 for all cubic polynomials $Q=x^{3}-\alpha x^{2}-\beta x-\gamma$ with $\gamma \neq 0$. The method of the proof is due to Bergmann [Ber78] and, even if simple, it is powerful (see [Ber93] for many interesting examples coming basically from rational homotopy theory).

Briefly, instead of having a semi-oriented graph as in Sect. 3 we shall work with an oriented graph and the relation $\leqq$ will be a total order, namely the lexicographic one. So we try to solve inductively all the ambiguities eventually adding new relations. We remark that this way all ambiguities are interactive ones so they could be listed. It remains to find the patience of checking all of them.

We proceed with 3 relations:

(1) $b_{2} b_{1} b_{2}=b_{1} b_{2} b_{1}$,

(2) $b_{1}^{3}=\alpha b_{1}^{2}+\beta b_{1}+\gamma$,

(3) $b_{2}^{3}=\alpha b_{2}^{2}+\beta b_{2}+\gamma$,

and the system of generators $S$ containing all words in $b_{1}$ and $b_{2}$ without subwords appearing in the left hand of some relation, i.e. without containing a $b_{2} b_{1} b_{2}, b_{1}^{3}, b_{2}^{3}$. Now we shall develop each ambiguity word (i.e. which has two resolutions) by underlining the subword replaced in each case. Away from the starting point the computations, even messy, became canonical, so the words have unique reduction, and we shall write only the final result. Also if an ambiguity is solvable, so that no new relation appears we mark by a $\square$ in the final space.

The interactions (2-2), (3-3), (1-2), (1-3) give only identities. Further relation,

(1-1) $b_{2} b_{1} \underline{b_{2} b_{1} b_{2}}=b_{2} b_{1}^{2} b_{2} b_{1}$ and $\underline{b_{2} b_{1} b_{2}} b_{1} b_{2}=b_{1} b_{2} b_{1}^{2} b_{2}$ so we obtain a new

$$
b_{2} b_{1}^{2} b_{2} b_{1}=b_{1} b_{2} b_{1}^{2} b_{2}
$$


Next we have an interaction

$$
\begin{aligned}
& \frac{b_{2} b_{1}^{2} b_{2} b_{1} b_{2}}{b_{2}}=b_{1} b_{2} b_{1}^{2} b_{2}^{2} \text { and } \\
& b_{2} b_{1}^{2} b_{2} b_{1} b_{2}=\alpha a b_{1} b_{2} b_{1}^{2} b_{2}+\beta b_{1} b_{2} b_{1}^{2}+\gamma b_{2}^{2} b_{1},
\end{aligned}
$$

and a new relation is obtained

$$
\begin{aligned}
& b_{1} b_{2} b_{1}^{2} b_{2}^{2}=\alpha b_{1} b_{2} b_{1}^{2} b_{2}+\beta b_{1} b_{2} b_{1}^{2}+\gamma b_{2}^{2} b_{1} . \\
& \underline{b_{2} b_{1}^{2} b_{2} b_{1}} b_{1}^{2}=\alpha b_{1}^{2} b_{2} b_{1}^{2} b_{2}+\beta b_{1} b_{2} b_{1}^{2} b_{2}+\gamma b_{2} b_{1}^{2} b_{2} \\
& b_{2} b_{1}^{2} b_{2} \underline{b_{1}^{3}}=\alpha b_{1}^{2} b_{2} b_{1}^{2} b_{2}+\beta b_{1} b_{2} b_{1}^{2} b_{2}+\gamma b_{2} b_{1}^{2} b_{2} . \\
& \underline{b_{2} b_{1} b_{2}} b_{1}^{2} b_{2} b_{1}=\alpha b_{1}^{2} b_{2} b_{1}^{2} b_{2}+\beta b_{1}^{2} b_{2} b_{1}^{2}+\gamma b_{1} b_{2}^{2} b_{1} \\
& b_{2} b_{1} b_{2} b_{1}^{2} b_{2} b_{1}=\alpha b_{1}^{2} b_{2} b_{1}^{2} b_{2}+\beta b_{1}^{2} b_{2} b_{1}^{2}+\gamma b_{1} b_{2}^{2} b_{1} \text {. }
\end{aligned}
$$

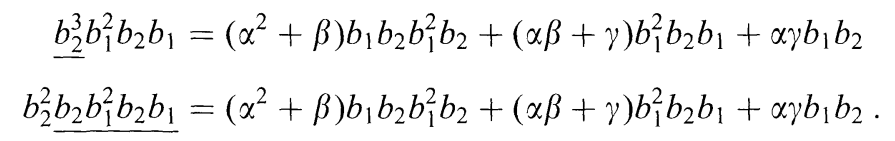

$$
\begin{aligned}
& \underline{b_{1}^{3}} b_{2} b_{1}^{2} b_{2}^{2}=\alpha^{2} b_{1}^{2} b_{2} b_{1}^{2} b_{2}+\alpha \beta b_{1}^{2} b_{2} b_{1}^{2}+\alpha \gamma b_{1} b_{2}^{2} b_{1}+\alpha \beta b_{1} b_{2} b_{1}^{2} b_{2} \\
& +\beta^{2} b_{1} b_{2} b_{1}^{2}+\beta \gamma b_{2}^{2} b_{1}+\gamma b_{2} b_{1}^{2} b_{2}^{2} \\
& b_{1}^{2} \underline{b_{1} b_{2} b_{1}^{2} b_{2}^{2}}=\alpha^{2} b_{1}^{2} b_{2} b_{1}^{2} b_{2}+\alpha \beta b_{1}^{2} b_{2} b_{1}^{2}+\alpha \gamma b_{2} b_{1}^{2} b_{2}+\alpha \beta b_{1} b_{2} b_{1}^{2} b_{2} \\
& +\beta^{2} b_{1} b_{2} b_{1}^{2}+\beta \gamma b_{2} b_{1}^{2}+\gamma b_{1}^{2} b_{2}^{2} b_{2} .
\end{aligned}
$$

Since we supposed $\gamma \neq 0$ we derive a new relation:

$$
b_{2} b_{1}^{2} b_{2}^{2}=b_{1}^{2} b_{2}^{2} b_{1}+\alpha\left(b_{2} b_{1}^{2} b_{2}-b_{1} b_{2}^{2} b_{1}\right)+\beta\left(b_{2} b_{1}^{2}-b_{2}^{2} b_{1}\right) .
$$

Now the left hand of (6) is included in the left hand of (5) and

$$
b_{1} \underline{b_{2} b_{1}^{2} b_{2}^{2}}=\alpha b_{1} b_{2} b_{1}^{2} b_{2}+\beta b_{1} b_{2} b_{1}^{2}+\gamma b_{2}^{2} b_{1} .
$$

So relation (5) is cancelled out when introducing (6).

$$
\begin{aligned}
\underline{b_{2} b_{1}^{2} b_{2}^{2} b_{2}=} & \alpha b_{2} b_{1}^{2} b_{2}^{2}+\alpha^{2}\left(b_{2} b_{1}^{2} b_{2}-b_{1} b_{2}^{2} b_{1}\right) \\
& +\alpha \beta\left(b_{2} b_{1}^{2}-b_{2}^{2} b_{1}\right)+\beta b_{2} b_{1}^{2} b_{2}+\gamma b_{2} b_{1}^{2} \\
b_{2} b_{1} \underline{b_{2}^{3}}= & \alpha b_{2} b_{1}^{2} b_{2}^{2}+\alpha^{2}\left(b_{2} b_{1}^{2} b_{2}-b_{1} b_{2}^{2} b_{1}\right)+\alpha \beta\left(b_{2} b_{1}^{2}-b_{2}^{2} b_{1}\right) \\
& +\beta b_{2} b_{1}^{2} b_{2}+\gamma b_{2} b_{1}^{2} .
\end{aligned}
$$

(6-1) $\quad \underline{b_{2} b_{1}^{2} b_{2}^{2}} b_{1} b_{2}=b_{1}^{2} b_{2}^{2} b_{1}^{2} b_{2}-\alpha b_{1} b_{2}^{2} b_{1}^{2} b_{2}-\beta b_{2}^{2} b_{1}^{2} b_{2}+\alpha \beta b_{2} b_{1}^{2} b_{2}$

$$
+\beta^{2} b_{1} b_{2} b_{1}+\beta \gamma b_{2}^{2}+\alpha^{2} b_{1} b_{2} b_{1}^{2} b_{2}+\alpha \beta b_{1} b_{2} b_{1}^{2}+\alpha \gamma b_{2}^{2} b_{1},
$$

$b_{2} b_{1}^{2} b_{2} \underline{b_{2} b_{1} b_{2}}=\alpha b_{1}^{2} b_{2} b_{1}^{2} b_{2}+\alpha \beta b_{1} b_{2} b_{1}^{2}+\beta^{2} b_{1} b_{2} b_{1}+\beta \gamma b_{1} b_{2}+\gamma b_{2}^{2} b_{1}^{2}$. 
So another relation must be added:

$$
\begin{aligned}
b_{1}^{2} b_{2}^{2} b_{1}^{2} b_{2}= & \alpha\left(b_{1}^{2} b_{2} b_{1}^{2} b_{2}+b_{1} b_{2}^{2} b_{1}^{2} b_{2}\right)+\beta b_{2}^{2} b_{1}^{2} b_{2}-\alpha^{2} b_{1} b_{2} b_{1}^{2} b_{2}+\gamma b_{2}^{2} b_{1}^{2} \\
& -\alpha \beta b_{2} b_{1}^{2} b_{2}-\alpha \gamma b_{2}^{2} b_{1}+\beta \gamma\left(b_{1} b_{2}-b_{2}^{2}\right),
\end{aligned}
$$

$$
\begin{aligned}
\underline{b_{2} b_{1} b_{2}} b_{1}^{2} b_{2}^{2}= & \alpha^{2} b_{1} b_{2} b_{1}^{2} b_{2}+\alpha \beta b_{1}^{2} b_{2} b_{1}+\alpha \gamma b_{1} b_{2}^{2}+\alpha \beta b_{1} b_{2} b_{1}^{2} \\
& +\beta^{2} b_{1} b_{2} b_{1}+\beta \gamma b_{1} b_{2}+\alpha \gamma b_{2}^{2} b_{1}+\beta \gamma b_{2} b_{1}+\gamma^{2} b_{1}, \\
b_{2} b_{1} \underline{b_{2} b_{1}^{2} b_{2}^{2}=} & \alpha^{2} b_{1} b_{2} b_{1}^{2} b_{2}+\alpha \beta b_{1}^{2} b_{2} b_{1}+\alpha \gamma b_{1} b_{2}^{2}+\alpha \beta b_{1} b_{2} b_{1}^{2} \\
& +\beta^{2} b_{1} b_{2} b_{1}+\beta \gamma b_{1} b_{2}+\alpha \gamma b_{2}^{2} b_{1}+\beta \gamma b_{2} b_{1}+\gamma^{2} b_{1} .
\end{aligned}
$$

$$
\begin{aligned}
\underline{b_{2}^{3} b_{1}^{2} b_{2}^{2}=} & \gamma b_{1}^{2} b_{2}^{2}+\alpha^{2} b_{2}^{2} b_{1}^{2} b_{2}+\beta b_{1}^{2} b_{2}^{2} b_{1}+\alpha \beta\left(b_{2} b_{1}^{2} b_{2}-b_{1} b_{2}^{2} b_{1}\right) \\
& +\beta^{2}\left(b_{2} b_{1}^{2}-b_{2}^{2} b_{1}\right)+\alpha b_{1}^{2} b_{2}^{2} b_{1}^{2}+\alpha^{2}\left(b_{1} b_{2} b_{1}^{2} b_{2}-b_{1} b_{2}^{2} b_{1}^{2}\right) \\
& -\alpha^{2} b_{1}^{2} b_{2} b_{1}^{2}-\alpha^{2} \beta b_{2}^{2} b_{1}-\alpha \beta \gamma b_{1}+\alpha^{2} \beta b_{2} b_{1}^{2}+\alpha \beta \gamma b_{2}, \\
b_{2}^{2} \underline{b_{2} b_{1}^{2} b_{2}^{2}=} & \gamma b_{1}^{2} b_{2}^{2}+\alpha^{2} b_{2}^{2} b_{1}^{2} b_{2}+\beta b_{1}^{2} b_{2}^{2} b_{1}+\alpha \beta\left(b_{2} b_{1}^{2} b_{2}-b_{1} b_{2}^{2} b_{1}\right) \\
& +\beta^{2}\left(b_{2} b_{1}^{2}-b_{2}^{2} b_{1}\right)+\alpha b_{1}^{2} b_{2}^{2} b_{1}^{2}+\alpha^{2}\left(b_{1} b_{2} b_{1}^{2} b_{2}-b_{1} b_{2}^{2} b_{1}^{2}\right) \\
& -\alpha^{2} b_{1}^{2} b_{2} b_{1}^{2}-\alpha^{2} \beta b_{2}^{2} b_{1}-\alpha \beta \gamma b_{1}+\alpha^{2} \beta b_{2} b_{1}^{2}+\alpha \beta \gamma b_{2} .
\end{aligned}
$$

$$
\begin{aligned}
b_{1} \underline{b_{1}^{2} b_{2}^{2} b_{1}^{2} b_{2}}= & \alpha b_{1}^{2} b_{2}^{2} b_{1}^{2} b_{2}+\beta b_{1} b_{2}^{2} b_{1}^{2} b_{2}+\gamma b_{1} b_{2}^{2} b_{1}^{2}+\alpha \gamma\left(b_{2} b_{1}^{2} b_{2}-b_{1} b_{2}^{2} b_{1}\right) \\
& +\beta \gamma\left(b_{1}^{2} b_{2}-b_{1} b_{2}^{2}\right)
\end{aligned}
$$

hence we get the last relation we used in Sect. 2, namely

$$
b_{2}^{2} b_{1}^{2} b_{2}=b_{1} b_{2}^{2} b_{1}^{2}+\alpha\left(b_{2} b_{1}^{2} b_{1}-b_{1} b_{2}^{2} b_{1}\right)+\beta\left(b_{1}^{2} b_{2}-b_{1} b_{2}^{2}\right) .
$$

Now using these eight relations the system of generators $S$ reduces to the 24 elements $e_{1}, e_{2}, \ldots, e_{24}$. So it remains to check that all ambiguities are solvable. and

Before proceeding we remark that the left hand of (8) is included in that of (7)

$$
\begin{aligned}
b_{1}^{2} \underline{b_{2}^{2} b_{1}^{2} b_{2}}= & \alpha b_{1}^{2} b_{2} b_{1}^{2} b_{2}+\alpha b_{1}^{2} b_{2}^{2} b_{1}^{2}+\beta b_{1} b_{2}^{2} b_{1}^{2}+\gamma b_{2}^{2} b_{1}^{2}-\alpha^{2} b_{1}^{2} b_{2}^{2} b_{1} \\
& -\alpha \beta b_{1} b_{2}^{2} b_{1}-\alpha \gamma b_{2}^{2} b_{1}+\left(\alpha^{2} \beta+\beta^{2}\right) b_{1}^{2} b_{2}+\left(\alpha \beta^{2}+\beta \gamma\right) b_{1} b_{2} \\
& +\alpha \beta \gamma b_{2}-\alpha \beta b_{1}^{2} b_{2}^{2}-\beta^{2} b_{1} b_{2}^{2}-\beta \gamma b_{2}^{2},
\end{aligned}
$$

so (7) is cancelled out.

$$
\begin{aligned}
\underline{b_{2} b_{1} b_{2}} b_{2} b_{1}^{2} b_{2} & =\alpha \beta b_{1}^{2} b_{2} b_{1}+\beta^{2} b_{1} b_{2} b_{1}+\beta \gamma b_{2} b_{1}, \\
b_{2} b_{1} b_{2}^{2} b_{1}^{2} b_{2} & =\alpha \beta b_{1}^{2} b_{2} b_{1}+\beta^{2} b_{1} b_{2} b_{1}+\beta \gamma b_{2} b_{1} .
\end{aligned}
$$


$(3-8)$

$$
\begin{aligned}
\underline{b_{2}^{3}} b_{1}^{2} b_{2} & =\alpha b_{1} b_{2}^{2} b_{1}^{2}+\alpha^{2}\left(b_{2} b_{1}^{2} b_{2}-b_{1} b_{2}^{2} b_{1}\right)+\alpha \beta\left(b_{1}^{2} b_{2}-b_{1} b_{2}^{2}\right) \\
b_{2} \underline{b_{2}^{2} b_{1}^{2} b_{2}} & =\alpha b_{1} b_{2}^{2} b_{1}^{2}+\alpha^{2}\left(b_{2} b_{1}^{2} b_{2}-b_{1} b_{2}^{2} b_{1}\right)+\alpha \beta\left(b_{1}^{2} b_{2}-b_{1} b_{2}^{2}\right)
\end{aligned}
$$

$(6-8)_{1} \quad b_{2}^{2} b_{1}^{2} b_{2} b_{2}=b_{1}^{2} b_{2}^{2} b_{1}^{2}+\alpha\left(b_{1} b_{2} b_{1}^{2} b_{2}-b_{1}^{2} b_{2} b_{1}^{2}\right)+\alpha^{2}\left(b_{2} b_{1}^{2} b_{2}-b_{1} b_{2}^{2} b_{1}\right)$

$$
\begin{gathered}
+\alpha \beta\left(b_{1}^{2} b_{2}+b_{2} b_{1}^{2}-b_{2}^{2} b_{1}-b_{1} b_{2}^{2}\right)+\beta \gamma\left(b_{2}-b_{1}\right) \\
b_{2} \underline{b_{2} b_{1}^{2} b_{2}^{2}}=b_{1}^{2} b_{2}^{2} b_{1}^{2}+\alpha\left(b_{1} b_{2} b_{1}^{2} b_{2}-b_{1}^{2} b_{2} b_{1}^{2}\right)+\alpha^{2}\left(b_{2} b_{1}^{2} b_{2}-b_{1} b_{2}^{2} b_{1}\right) \\
+\alpha \beta\left(b_{1}^{2} b_{2}+b_{2} b_{1}^{2}-b_{2}^{2} b_{1}-b_{1} b_{2}^{2}\right)+\beta \gamma\left(b_{2}-b_{1}\right) .
\end{gathered}
$$

$(6-8)_{2} \quad \underline{b_{2} b_{1}^{2} b_{2}} b_{1}^{2} b_{2}^{2}=2 \alpha^{2} b_{1}^{2} b_{2} b_{1}^{2} b_{2}+\alpha \beta b_{1}^{2} b_{2} b_{1}^{2}-\alpha^{3} b_{1} b_{2} b_{1}^{2} b_{2}$

$$
\begin{aligned}
& +\alpha \gamma\left(b_{1}^{2} b_{2}^{2}+b_{2}^{2} b_{1}^{2}+b_{1} b_{2}^{2} b_{1}\right)+\beta^{2} b_{2} b_{1}^{2} b_{2} \\
& +\beta \gamma\left(b_{1}^{2} b_{2}+b_{2} b_{1}^{2}\right)-\alpha^{2} \gamma\left(b_{1} b_{2}^{2}+b_{2}^{2} b_{1}\right) \\
& +\left(\alpha \beta^{2}+\beta \gamma\right) b_{1} b_{2} b_{1}+\gamma^{2} b_{1}^{2}-\alpha \beta \gamma b_{2}^{2}-\alpha \gamma^{2} b_{1} \\
& -\beta^{2} \gamma b_{2}-\beta \gamma^{2} \\
b_{2} b_{1}^{2} \underline{b_{2}^{2} b_{1}^{2} b_{2}}= & 2 \alpha^{2} b_{1}^{2} b_{2} b_{1}^{2} b_{2}+\alpha \beta b_{1}^{2} b_{2} b_{1}^{2}-\alpha^{3} b_{1} b_{2} b_{1}^{2} b_{2}
\end{aligned}
$$$$
+\alpha \gamma\left(b_{1}^{2} b_{2}^{2}+b_{2}^{2} b_{1}^{2}+b_{1} b_{2}^{2} b_{1}\right)+\beta^{2} b_{2} b_{1}^{2} b_{2}
$$$$
+\beta \gamma\left(b_{1}^{2} b_{2}+b_{2} b_{1}^{2}\right)-\alpha^{2} \gamma\left(b_{1} b_{2}^{2}+b_{2}^{2} b_{1}\right)
$$$$
+\left(\alpha \beta^{2}+\beta \gamma\right) b_{1} b_{2} b_{1}+\gamma^{2} b_{1}^{2}-\alpha \beta \gamma b_{2}^{2}-\alpha \gamma^{2} b_{1}
$$$$
-\beta^{2} \gamma b_{2}-\beta \gamma^{2} \text {. }
$$

(4-4) $\quad \underline{b_{2} b_{1}^{2} b_{2} b_{1}} b_{1} b_{2} b_{1}=\alpha^{2} b_{1}^{2} b_{2} b_{1}^{2} b_{2}+\alpha \beta b_{1} b_{2} b_{1}^{2} b_{2}+\alpha \beta b_{1}^{2} b_{2} b_{1}^{2}+\gamma b_{1} b_{2}^{2} b_{1}^{2}$

$$
\begin{aligned}
& +\beta^{2} b_{1}^{2} b_{2} b_{1}+\alpha \gamma b_{2} b_{1}^{2} b_{2}+\beta \gamma b_{1}^{2} b_{2}, \\
b_{2} b_{1}^{2} \underline{b_{2} b_{1}^{2} b_{2} b_{1}}= & \alpha^{2} b_{1}^{2} b_{2} b_{1}^{2} b_{2}+\alpha \beta b_{1} b_{2} b_{1}^{2} b_{2}+\alpha \beta b_{1}^{2} b_{2} b_{1}^{2}+\gamma b_{1} b_{2}^{2} b_{1}^{2} \\
& +\beta^{2} b_{1}^{2} b_{2} b_{1}+\alpha \gamma b_{2} b_{1}^{2} b_{2}+\beta \gamma b_{1}^{2} b_{2} .
\end{aligned}
$$

(4-6) $\quad b_{2} b_{1}^{2} b_{2} b_{1} b_{1} b_{2}^{2}=\left(\alpha^{2}+\beta\right) b_{1}^{2} b_{2} b_{1}^{2} b_{2}+(\alpha \beta+\gamma) b_{1}^{2} b_{2} b_{1}^{2}+\alpha \gamma b_{1} b_{2}^{2} b_{1}$ ，

$$
b_{2} b_{1}^{2} \underline{b_{2} b_{1}^{2} b_{2}^{2}}=\left(\alpha^{2}+\beta\right) b_{1}^{2} b_{2} b_{1}^{2} b_{2}+(\alpha \beta+\gamma) b_{1}^{2} b_{2} b_{1}^{2}+\alpha \gamma b_{1} b_{2}^{2} b_{1} .
$$

(6-4) $\quad \underline{b_{2} b_{1}^{2} b_{2}} b_{2} b_{1}^{2} b_{2} b_{1}=\alpha^{3} b_{1}^{2} b_{2} b_{1}^{2} b_{2}+\alpha^{2} \beta b_{1}^{2} b_{2} b_{1}^{2}+\left(2 \alpha^{2} \beta+\beta^{2}\right) b_{1} b_{2} b_{1}^{2} b_{2}$

$$
\begin{aligned}
& +\alpha \gamma\left(b_{1}^{2} b_{2}^{2} b_{1}+b_{1} b_{2}^{2} b_{1}^{2}\right)-\alpha^{2} \gamma b_{1} b_{2}^{2} b_{1} \\
& +\left(\alpha \beta^{2}+\beta \gamma\right) b_{1}^{2} b_{2} b_{1}+\left(\beta \gamma+\alpha \beta^{2}\right) b_{1} b_{2} b_{1}^{2} \\
& +2 \alpha^{2} \gamma b_{2} b_{1}^{2} b_{2}+\alpha \beta \gamma\left(b_{1}^{2} b_{2}+b_{2} b_{1}^{2}\right) \\
& +\alpha \gamma^{2} b_{2}^{2}+\beta \gamma^{2} b_{2}+\gamma^{3},
\end{aligned}
$$




$$
\begin{aligned}
b_{2} b_{1}^{2} b_{2} \underline{b_{2} b_{1}^{2} b_{2} b_{1}=} & \alpha^{3} b_{1}^{2} b_{2} b_{1}^{2} b_{2}+\alpha^{2} \beta b_{1}^{2} b_{2} b_{1}^{2}+\left(2 \alpha^{2} \beta+\beta^{2}\right) b_{1} b_{2} b_{1}^{2} b_{2} \\
& +\alpha \gamma\left(b_{1}^{2} b_{2}^{2} b_{1}+b_{1} b_{2}^{2} b_{1}^{2}\right)-\alpha^{2} \gamma b_{1} b_{2}^{2} b_{1} \\
& +\left(\alpha \beta^{2}+\beta \gamma\right) b_{1}^{2} b_{2} b_{1}+\left(\beta \gamma+\alpha \beta^{2}\right) b_{1} b_{2} b_{1}^{2} \\
& +2 \alpha^{2} \gamma b_{2} b_{1}^{2} b_{2}+\alpha \beta \gamma\left(b_{1}^{2} b_{2}+b_{2} b_{1}^{2}\right) \\
& +\alpha \gamma^{2} b_{2}^{2}+\beta \gamma^{2} b_{2}+\gamma^{3} .
\end{aligned}
$$

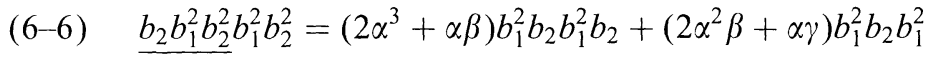

$$
\begin{aligned}
& +\beta^{2} b_{1}^{2} b_{2}^{2} b_{1}-\alpha^{4} b_{1} b_{2} b_{1}^{2} b_{2}+\alpha \gamma b_{1} b_{2}^{2} b_{1}^{2} \\
& +\left(\alpha \beta^{2}+\beta \gamma\right) b_{1}^{2} b_{2} b_{1}+\left(\alpha^{2} \gamma+\beta \gamma\right) b_{1}^{2} b_{2}^{2} \\
& -\left(\alpha^{3} \beta+\alpha^{2} \gamma\right) b_{1} b_{2} b_{1}^{2}+\left(\alpha^{2} \gamma-\alpha \beta^{2}\right) b_{1} b_{2}^{2} b_{1} \\
& +\left(\alpha \beta^{2}+\alpha^{2} \gamma+\beta \gamma\right) b_{2} b_{1}^{2} b_{2}+\left(2 \alpha \beta \gamma+\gamma^{2}\right) b_{1}^{2} b_{2} \\
& -\left(\alpha^{3} \gamma+\alpha \beta \gamma\right) b_{1} b_{2}^{2}+\beta^{3} b_{2} b_{1}^{2}-\left(\alpha^{3} \gamma+\beta^{3}\right) b_{2}^{2} b_{1}+\alpha \gamma^{2} b_{1}^{2} \\
& -\left(\alpha^{2} \beta \gamma+\alpha \gamma^{2}\right) b_{1} b_{2}-\left(\alpha^{2} \beta \gamma+\beta^{2} \gamma\right) b_{2}^{2}-\alpha^{2} \gamma^{2} b_{1} \\
& +\left(\alpha \beta^{2} \gamma+\beta \gamma^{2}\right) b_{2}-\alpha \beta \gamma^{2} .
\end{aligned}
$$

$$
\begin{aligned}
b_{2} b_{1}^{2} b_{2} b_{2} b_{1}^{2} b_{2}^{2}= & \left(2 \alpha^{3}+\alpha \beta\right) b_{1}^{2} b_{2} b_{1}^{2} b_{2}+\left(2 \alpha^{2} \beta+\alpha \gamma\right) b_{1}^{2} b_{2} b_{1}^{2} \\
& +\beta^{2} b_{1}^{2} b_{2}^{2} b_{1}-\alpha^{4} b_{1} b_{2} b_{1}^{2} b_{2}+\alpha \gamma b_{1} b_{2}^{2} b_{1}^{2} \\
& +\left(\alpha \beta^{2}+\beta \gamma\right) b_{1}^{2} b_{2} b_{1}+\left(\alpha^{2} \gamma+\beta \gamma\right) b_{1}^{2} b_{2}^{2} \\
& -\left(\alpha^{3} \beta+\alpha^{2} \gamma\right) b_{1} b_{2} b_{1}^{2}+\left(\alpha^{2} \gamma-\alpha \beta^{2}\right) b_{1} b_{2}^{2} b_{1} \\
& +\left(\alpha \beta^{2}+\alpha^{2} \gamma+\beta \gamma\right) b_{2} b_{1}^{2} b_{2}+\left(2 \alpha \beta \gamma+\gamma^{2}\right) b_{1}^{2} b_{2} \\
& -\left(\alpha^{3} \gamma+\alpha \beta \gamma\right) b_{1} b_{2}^{2}+\beta^{3} b_{2} b_{1}^{2}-\left(\alpha^{3} \gamma+\beta^{3}\right) b_{2}^{2} b_{1}+\alpha \gamma^{2} b_{1}^{2} \\
& -\left(\alpha^{2} \beta \gamma+\alpha \gamma^{2}\right) b_{1} b_{2}-\left(\alpha^{2} \beta \gamma+\beta^{2} \gamma\right) b_{2}^{2}-\alpha^{2} \gamma^{2} b_{1} \\
& +\left(\alpha \beta^{2} \gamma+\beta \gamma^{2}\right) b_{2}-\alpha \beta \gamma^{2} .
\end{aligned}
$$

$$
\underline{b_{2}^{2} b_{1}^{2} b_{2}} b_{1} b_{2}=\alpha \gamma b_{1} b_{2}^{2}+\beta \gamma b_{1} b_{2}+\gamma^{2} b_{1},
$$$$
b_{2}^{2} b_{1}^{2} \underline{b_{2} b_{1} b_{2}}=\alpha \gamma b_{1} b_{2}^{2}+\beta \gamma b_{1} b_{2}+\gamma^{2} b_{1} \text {. }
$$

$$
\begin{aligned}
\underline{b_{2}^{2} b_{1}^{2} b_{2}} b_{2}^{2}= & \alpha b_{1}^{2} b_{2}^{2} b_{1}^{2}+\alpha^{2}\left(b_{1} b_{2} b_{1}^{2}-b_{1}^{2} b_{2} b_{1}^{2}\right) \\
& +\left(\alpha^{3}+\alpha \beta\right)\left(b_{2} b_{1}^{2} b_{2}-b_{1} b_{2}^{2} b_{1}\right)+\alpha^{2} \beta\left(b_{2} b_{1}^{2}-b_{2}^{2} b_{1}\right) \\
& +\left(\alpha^{2} \beta+\beta^{2}\right)\left(b_{1}^{2} b_{2}-b_{1} b_{2}^{2}\right) \\
& +\beta b_{1} b_{2}^{2} b_{1}^{2}+\alpha \beta \gamma\left(b_{2}-b_{1}\right),
\end{aligned}
$$




$$
\begin{aligned}
b_{2}^{2} b_{1}^{2} \underline{b_{2}^{3}=} & \alpha b_{1}^{2} b_{2}^{2} b_{1}^{2}+\alpha^{2}\left(b_{1} b_{2} b_{1}^{2}-b_{1}^{2} b_{2} b_{1}^{2}\right) \\
& +\left(\alpha^{3}+\alpha \beta\right)\left(b_{2} b_{1}^{2} b_{2}-b_{1} b_{2}^{2} b_{1}\right)+\alpha^{2} \beta\left(b_{2} b_{1}^{2}-b_{2}^{2} b_{1}\right) \\
& +\left(\alpha^{2} \beta+\beta^{2}\right)\left(b_{1}^{2} b_{2}-b_{1} b_{2}^{2}\right) \\
& +\beta b_{1} b_{2}^{2} b_{1}^{2}+\alpha \beta \gamma\left(b_{2}-b_{1}\right)
\end{aligned}
$$

$$
\begin{aligned}
\underline{b_{2}^{2} b_{1}^{2} b_{2} b_{1}} & =\alpha b_{1} b_{2} b_{1}^{2} b_{2}+\beta b_{1}^{2} b_{2} b_{1}+\gamma b_{1} b_{2}^{2}, \\
b_{2} b_{2} b_{1}^{2} b_{2} b_{1} & =\alpha b_{1} b_{2} b_{1}^{2} b_{2}+\beta b_{1}^{2} b_{2} b_{1}+\gamma b_{1} b_{2}^{2} .
\end{aligned}
$$

$(8-4)_{2}$

$$
\begin{aligned}
\underline{b_{2}^{2} b_{1}^{2} b_{2} b_{1}^{2} b_{2} b_{1}=} & \left(\alpha^{3}+\alpha \beta\right) b_{1}^{2} b_{2} b_{1}^{2} b_{2}+\left(\alpha^{2} \beta+\alpha \gamma\right) b_{1}^{2} b_{2} b_{1}^{2} \\
& +\left(\alpha^{2} \beta+\beta^{2}\right) b_{1} b_{2} b_{1}^{2} b_{2}+\alpha \gamma b_{1} b_{2}^{2} b_{1}^{2} \\
& +\left(\alpha^{2} \gamma+\beta \gamma\right) b_{2} b_{1}^{2} b_{2}+\left(\alpha \beta^{2}+\beta \gamma\right) b_{1}^{2} b_{2} b_{1} \\
& +\left(\alpha \beta \gamma+\gamma^{2}\right) b_{1}^{2} b_{2}, \\
b_{2}^{2} b_{1}^{2} \underline{b_{2} b_{1}^{2} b_{2} b_{1}=} & \left(\alpha^{3}+\alpha \beta\right) b_{1}^{2} b_{2} b_{1}^{2} b_{2}+\left(\alpha^{2} \beta+\alpha \gamma\right) b_{1}^{2} b_{2} b_{1}^{2} \\
& +\left(\alpha^{2} \beta+\beta^{2}\right) b_{1} b_{2} b_{1}^{2} b_{2}+\alpha \gamma b_{1} b_{2}^{2} b_{1}^{2} \\
& +\left(\alpha^{2} \gamma+\beta \gamma\right) b_{2} b_{1}^{2} b_{2}+\left(\alpha \beta^{2}+\beta \gamma\right) b_{1}^{2} b_{2} b_{1} \\
& +\left(\alpha \beta \gamma+\gamma^{2}\right) b_{1}^{2} b_{2} .
\end{aligned}
$$

$(8-6) \quad b_{2}^{2} b_{1}^{2} b_{2} b_{1}^{2} b_{2}^{2}=\left(\alpha^{3}+2 \alpha \beta+\gamma\right) b_{1}^{2} b_{2} b_{1}^{2} b_{2}+\left(\alpha^{2} \beta+\alpha \gamma+\beta^{2}\right) b_{1}^{2} b_{2} b_{1}^{2}$

$$
+\left(\alpha^{2} \gamma+\beta \gamma\right) b_{1} b_{2}^{2} b_{1}
$$

$$
\begin{aligned}
b_{2}^{2} b_{1}^{2} \underline{b_{2} b_{1}^{2} b_{2}^{2}}= & \left(\alpha^{3}+2 \alpha \beta+\gamma\right) b_{1}^{2} b_{2} b_{1}^{2} b_{2}+\left(\alpha^{2} \beta+\alpha \gamma+\beta^{2}\right) b_{1}^{2} b_{2} b_{1}^{2} \\
& +\left(\alpha^{2} \gamma+\beta \gamma\right) b_{1} b_{2}^{2} b_{1} .
\end{aligned}
$$

$$
\begin{aligned}
\underline{b_{2}^{2} b_{1}^{2} b_{2} b_{2} b_{1}^{2} b_{2}=} & \left(2 \alpha^{3}+\alpha \beta\right) b_{1}^{2} b_{2} b_{1}^{2} b_{2}+\left(2 \alpha^{2} \beta+\alpha \gamma\right) b_{1}^{2} b_{2} b_{1}^{2} \\
& +\alpha \gamma b_{1}^{2} b_{2}^{2} b_{1}+\beta^{2} b_{1} b_{2}^{2} b_{1}^{2}-\alpha^{4} b_{1} b_{2} b_{1}^{2} b_{2} \\
& -\left(\alpha^{3} \beta+\alpha^{2} \gamma\right) b_{1}^{2} b_{2} b_{1}+\left(\alpha \beta^{2}+\beta \gamma\right) b_{1} b_{2} b_{1}^{2} \\
& +\left(\alpha^{2} \gamma-\alpha \beta^{2}\right) b_{1} b_{2}^{2} b_{1}+\left(\alpha \beta^{2}+\beta \gamma+\alpha^{2} \gamma\right) b_{2} b_{1}^{2} b_{2} \\
& +\left(\alpha^{2} \gamma+\beta \gamma\right) b_{2}^{2} b_{1}^{2}+\beta^{3} b_{1}^{2} b_{2}-\left(\alpha^{3} \gamma+\beta^{3}\right) b_{1} b_{2}^{2} \\
& +\left(2 \alpha \beta \gamma+\gamma^{2}\right) b_{2} b_{1}^{2}-\left(\alpha \beta \gamma+\alpha^{3} \gamma\right) b_{2}^{2} b_{1}+\alpha \gamma^{2} b_{1}^{2} \\
& -\left(\alpha^{2} \beta \gamma+\alpha \gamma^{2}\right) b_{2} b_{1}-\left(\alpha^{2} \beta \gamma+\beta^{2} \gamma\right) b_{2}^{2} \\
& -\alpha^{2} \gamma^{2} b_{1}-\left(\alpha \beta^{2} \gamma+\beta \gamma^{2}\right) b_{2}-\alpha \beta \gamma^{2},
\end{aligned}
$$




$$
\begin{aligned}
b_{2}^{2} b_{1}^{2} \underline{b_{2}^{2} b_{1}^{2} b_{2}=} & \left(2 \alpha^{3}+\alpha \beta\right) b_{1}^{2} b_{2} b_{1}^{2} b_{2}+\left(2 \alpha^{2} \beta+\alpha \gamma\right) b_{1}^{2} b_{2} b_{1}^{2} \\
& +\alpha \gamma b_{1}^{2} b_{2}^{2} b_{1}+\beta^{2} b_{1} b_{2}^{2} b_{1}^{2}-\alpha^{4} b_{1} b_{2} b_{1}^{2} b_{2} \\
& -\left(\alpha^{3} \beta+\alpha^{2} \gamma\right) b_{1}^{2} b_{2} b_{1}+\left(\alpha \beta^{2}+\beta \gamma\right) b_{1} b_{2} b_{1}^{2} \\
& +\left(\alpha^{2} \gamma-\alpha \beta^{2}\right) b_{1} b_{2}^{2} b_{1}+\left(\alpha \beta^{2}+\beta \gamma+\alpha^{2} \gamma\right) b_{2} b_{1}^{2} b_{2} \\
& +\left(\alpha^{2} \gamma+\beta \gamma\right) b_{2}^{2} b_{1}^{2}+\beta^{3} b_{1}^{2} b_{2}-\left(\alpha^{3} \gamma+\beta^{3}\right) b_{1} b_{2}^{2} \\
& +\left(2 \alpha \beta \gamma+\gamma^{2}\right) b_{2} b_{1}^{2}-\left(\alpha \beta \gamma+\alpha^{3} \gamma\right) b_{2}^{2} b_{1}+\alpha \gamma^{2} b_{1}^{2} \\
& -\left(\alpha^{2} \beta \gamma+\alpha \gamma^{2}\right) b_{2} b_{1}-\left(\alpha^{2} \beta \gamma+\beta^{2} \gamma\right) b_{2}^{2} \\
& -\alpha^{2} \gamma^{2} b_{1}-\left(\alpha \beta^{2} \gamma+\beta \gamma^{2}\right) b_{2}-\alpha \beta \gamma^{2} .
\end{aligned}
$$

This ends the proof of Proposition 2.1.

\section{B. Appendix: The Quotient $K_{3}$}

We get below the complete proof of Proposition 2.10.

Since the dimension of $P(3)=H(Q, 3) / R(\mu)$ is 21 the following relations will become identities in $P(3)$ :

$$
\begin{gathered}
b_{1} b_{2} b_{1}^{2} b_{2}=b_{2} b_{1}^{2} b_{2} b_{1}, \\
b_{2} R(\mu)=0 .
\end{gathered}
$$

These equations are written in implicit form. We must express all factors in terms of the basis elements from $B-B^{\prime}$ of the 21 dimensional module. We have seen that $R(\mu)$ may be reduced to a simpler relation

$$
b_{2} b_{1}^{2} b_{2}=\sum_{i, j} \lambda_{l j} b_{1}^{l} b_{2} b_{1}^{\prime}+\mu_{l j} b_{1}^{i} b_{2}^{2} b_{1}^{j}+\sum_{i} v_{i} b_{1}^{i} .
$$

Lemma B.1. Equation (1) has the following solutions:

$$
\begin{aligned}
& \mu_{i j}=\mu_{j 1} \text { and } \lambda_{i j}=\lambda_{j i} \text { for all } i, j, \\
& \mu_{00}=\mu_{12}-\beta \mu_{02} ; \mu_{22}=\mu_{01}+\alpha \mu_{02} ; \mu_{11}=(\alpha \beta+1) \mu_{02}+\beta \mu_{01}-\alpha \mu_{12}, \\
& \lambda_{00}=\lambda_{12}-\beta \lambda_{02} ; \lambda_{22}=\lambda_{01}+\alpha \lambda_{02} ; \lambda_{11}=(\alpha \beta+1) \lambda_{02}+\beta \lambda_{01}-\alpha \lambda_{12} .
\end{aligned}
$$

Proof. We have

$$
\begin{aligned}
b_{1} b_{2} b_{1}^{2} b_{2}= & \sum_{i j} \lambda_{i j} b_{1}^{l+1} b_{2} b_{1}^{k}+\mu_{i j} b_{1}^{l+1} b_{2}^{2} b_{1}^{k}+\sum_{i} v_{i} b_{1}^{i+1} \\
= & \gamma \lambda_{02} b_{2}+\left(\lambda_{00}+\beta \lambda_{02}\right) b_{1} b_{2}+\gamma \lambda_{21} b_{2} b_{1}+\left(\lambda_{01}+\beta \lambda_{12}\right) b_{1} b_{2} b_{1} \\
& +\left(\lambda_{02}+\beta \lambda_{22}\right) b_{1} b_{2} b_{1}^{2}+\left(\lambda_{01}+\alpha \lambda_{02}\right) b_{1}^{2} b_{2} \\
& +\gamma \lambda_{22} b_{2} b_{1}^{2}+\left(\lambda_{11}+\alpha \lambda_{12}\right) b_{1}^{2} b_{2} b_{1}+\left(\lambda_{12}+\alpha \lambda_{22}\right) b_{1}^{2} b_{2} b_{1}^{2} \\
& +\gamma \mu_{02} b_{2}^{2}+\left(\mu_{00}+\beta \mu_{02}\right) b_{1} b_{2}^{2}+\gamma \mu_{21} b_{2}^{2} b_{1}-\left(\mu_{01}+\beta \mu_{12}\right) b_{1} b_{2}^{2} b_{1}
\end{aligned}
$$




$$
\begin{aligned}
& +\left(\mu_{02}+\beta \mu_{22}\right) b_{1} b_{2}^{2} b_{1}^{2}+\left(\mu_{01}+\alpha \mu_{02}\right) b_{1}^{2} b_{2}^{2} \\
& +\gamma \mu_{22} b_{2}^{2} b_{1}^{2}+\left(\mu_{11}+\alpha \mu_{12}\right) b_{1}^{2} b_{2}^{2} b_{1}+\left(\mu_{12}+\alpha \mu_{22}\right) b_{1}^{2} b_{2}^{2} b_{1}^{2} \\
& +\gamma v_{2}+\left(v_{0}+\beta v_{2}\right) b_{1}+\left(v_{1}+\alpha v_{2}\right) b_{1}^{2},
\end{aligned}
$$

and a similar expression (the symmetric one) may be written for $b_{2} b_{1}^{2} b_{2} b_{1}$. By comparison of these expressions and using the hypothesis (that the basis of $L_{3}$ descends to one of $P(3)$ ) we find the relations stated above.

In the same manner we can write the system of equations derived from (2). After we used the reductions from Lemma 2.9 we obtain the following quadratic system (S): Set $c_{1}=\lambda_{02}+\alpha \mu_{02} ; c_{2}=\lambda_{12}+\alpha \mu_{12} ; c_{3}=\lambda_{22}+\alpha \mu_{22}$,

$$
\begin{aligned}
& 0=v_{0}+c_{3} \lambda_{01}+\left(c_{2}+\alpha c_{3}-\beta c_{1}+\alpha \beta\right) \lambda_{02}+\left(c_{1}-\alpha\right) \lambda_{12} \\
& +\alpha \beta \mu_{01}+\beta\left(\alpha^{2}-\beta\right) \mu_{02}+2 \beta \mu_{12}, \\
& 0=\left(c_{1}-\alpha+\beta c_{3}\right) \lambda_{01}+(\alpha \beta+1) c_{3} \lambda_{02}+\left(c_{2}+1\right) \lambda_{12}, \\
& 0=v_{1}+\left(c_{1}-\alpha+\beta c_{3}\right) \lambda_{01}+(\alpha \beta+1) c_{3} \lambda_{02}+c_{2} \lambda_{12} \\
& +\beta(\alpha \beta+2) \mu_{01}+\alpha \beta(\alpha \beta+1) \mu_{02}+\beta^{2} \mu_{12}, \\
& 0=\left(1-\alpha \beta+\beta c_{1}+c_{2}+\beta^{2} c_{3}\right) \lambda_{01}+(\alpha \beta+1)\left(c_{1}+\beta c_{3}-\alpha\right) \lambda_{02} \\
& +\left(\beta+\alpha^{2}-\alpha c_{1}+\beta c_{2}-\alpha \beta c_{3}\right) \lambda_{12}, \\
& 0=\left(\beta+\left(\beta^{2}+\alpha \beta+1\right) c_{3}\right) \lambda_{01}+\left(\alpha \beta+1+c_{2}+(\alpha+\beta)(\alpha \beta+1) c_{3}\right) \lambda_{02} \\
& +\left(c_{1}+\beta c_{2}-\alpha \beta c_{3}-\alpha\right) \lambda_{12}, \\
& 0=\left(c_{2}+\alpha c_{3}\right) \lambda_{01}+\left(c_{1}+\alpha c_{2}+\alpha^{2} c_{3}-\alpha\right) \lambda_{02}+c_{3} \lambda_{12}+\mu_{12}-\beta, \\
& 0=v_{2}+\left(\left(c_{2}+\alpha c_{3}\right) \lambda_{01}+\left(c_{1}+\alpha c_{2}+\alpha^{2} c_{3}-\alpha\right) \lambda_{02}+c_{3} \lambda_{12}\right. \\
& +\beta\left(\alpha^{2}+\beta\right) \mu_{01}+\left(\alpha \beta\left(\alpha^{2}+\beta\right)+2 \beta\right) \mu_{02}+\alpha \beta \mu_{12}, \\
& 0=\left(\beta c_{2}+(\alpha \beta+1) c_{3}\right) \lambda_{01}+\left((\alpha \beta+1) c_{2}+\alpha(\alpha \beta+1-\alpha) c_{3}\right) \lambda_{02} \\
& +\left(c_{1}+\left(\alpha^{2}+\beta\right) c_{3}-\alpha\right) \lambda_{12}+\mu_{01}+\beta \mu_{12}, \\
& 0=\left(c_{1}+\alpha c_{2}+\left(\alpha^{2}+\beta\right) c_{3}-\alpha \beta\right) \lambda_{01} \\
& \left.+\left(\alpha c_{1}+\alpha^{2} c_{2}+\left(\alpha\left(\alpha^{2}+\beta\right)+1\right)\right) c_{3}-\alpha(\alpha \beta+1)\right) \lambda_{02} \\
& +\left(c_{2}+\alpha c_{3}+\alpha^{2}\right) \lambda_{12}+(\alpha \beta+1) \mu_{02}+\beta \mu_{01}, \\
& 0=\lambda_{12}-\beta \lambda_{02}+\left(c_{3}-\beta\right) \mu_{01}+\left(c_{2}-\beta c_{1}+\alpha c_{3}-\alpha \beta\right) \mu_{02}+c_{1} \mu_{12}, \\
& -\beta=\left(c_{1}+\beta c_{3}-2 \alpha\right) \mu_{01}+\left((\alpha \beta+1) c_{3}-\alpha^{2}\right) \mu_{02}+c_{2} \mu_{12}, \\
& 0=\lambda_{01}+\left(c_{1}+\beta c_{3}-\beta^{2}\right) \mu_{01}+(\alpha \beta+1)\left(c_{3}-\beta\right) \mu_{02}+c_{2} \mu_{12},
\end{aligned}
$$




$$
\begin{aligned}
-\alpha= & \left(\beta c_{1}+c_{2}+\beta^{2} c_{3}-2 \alpha \beta\right) \mu_{01}+(\alpha \beta+1)\left(c_{1}+\beta c_{3}-2 \alpha\right) \mu_{02} \\
& +\left(\beta c_{2}-\alpha c_{1}+c_{3}+\alpha^{2}\right) \mu_{12} \\
1= & \left(\beta c_{2}+(\alpha \beta+1) c_{3}-\alpha^{2}\right) \mu_{01}+\left((\alpha \beta+1) c_{2}+\alpha(\alpha \beta+1) c_{3}-\alpha^{3}\right) \mu_{02} \\
& +\left(c_{1}+\beta c_{3}-2 \alpha\right) \mu_{12} \\
0= & \left(c_{2}+\alpha c_{3}+1\right) \mu_{01}+\left(c_{1}+\alpha c_{2}+\alpha^{2} c_{3}\right) \mu_{02}+c_{3} \mu_{12} \\
0= & \lambda_{12}+\left(c_{2}+\alpha c_{3}-\alpha \beta\right) \mu_{01}+\left(c_{1}+\alpha c_{2}+\alpha^{2} c_{3}-\alpha^{2} \beta\right) \mu_{02} \\
& +\left(c_{3}-\beta\right) \mu_{12}, \\
0= & \left(\beta c_{2}+(\alpha \beta+1) c_{3}+\beta\right) \mu_{01}+(\alpha \beta+1)\left(c_{2}+\alpha c_{3}+1\right) \mu_{02} \\
& +\left(c_{1}+(\beta-\alpha) c_{3}\right) \mu_{12}, \\
0= & \left(c_{1}+\alpha c_{2}+\left(\alpha^{2}+\beta\right) c_{3}\right) \mu_{01}+\alpha\left(c_{1}+\alpha c_{2}+\left(\alpha^{2}+\beta\right) c_{3}\right) \mu_{02} \\
& +\left(c_{2}+\alpha c_{3}+1\right) \mu_{12}, \\
0= & c_{3} v_{0}+\left(c_{2}+\alpha c_{3}+1\right) v_{1}+\left(c_{1}+\alpha c_{2}+\left(\alpha^{2}+\beta\right) c_{3}-\alpha\right) v_{2}+\mu_{02} \\
0= & c_{2} v_{0}+\left(c_{1}+\beta c_{3}-\alpha\right) v_{1}+\left(\beta c_{2}+(\alpha \beta+1) c_{3}\right) v_{2}+\mu_{01}, \\
0= & \left(c_{1}-\alpha\right) v_{0}+c_{3} v_{1}+\left(c_{2}+\alpha c_{3}\right) v_{2}+\mu_{12}-\beta \mu_{02}=0 .
\end{aligned}
$$

We are able now to prove the first part of the proposition. Remark that for $\alpha=\beta=0$, we obtain a unique solution for (S) namely:

$$
\begin{aligned}
& \mu_{01}^{0}=\mu_{12}^{0}=0 ; \quad \mu_{02}^{0}=-1 ; \quad \lambda_{01}^{0}=\lambda_{02}^{0}=0 ; \\
& \lambda_{12}^{0}=-1 ; \quad v_{0}^{0}=v_{2}^{0}=0 ; \quad v_{1}^{0}=-1 .
\end{aligned}
$$

What it remains to show is that this solution gives indeed a quotient of dimension 21. So let $Q$ be $X^{3}-1$ from now on. Let $I$ be the ideal generated by the relation

$$
R_{0}=b_{2} b_{1}^{2} b_{2}+b_{1} b_{2}^{2} b_{1}+b_{1}^{2} b_{2} b_{1}+b_{1} b_{2} b_{1}^{2}+b_{1}^{2} b_{2}^{2}+b_{2}^{2} b_{1}^{2}+b_{1}+b_{2} \text {. }
$$

Set also

$$
\begin{aligned}
& R_{1}=b_{1} b_{2} b_{1}^{2} b_{2}+b_{1}^{2} b_{2} b_{1}^{2}+b_{1} b_{2}^{2} b_{1}^{2}+b_{1}^{2} b_{2}^{2} b_{1}+B_{1} b_{2}+b_{2} b_{1}+b_{1}^{2}+b_{2}^{2}, \\
& R_{2}=b_{1}^{2} b_{2} b_{1}^{2} b_{2}+b_{1}^{2} b_{2}^{2} b_{1}^{2}+b_{1}^{2} b_{2}+b_{2} b_{1}^{2}+b_{1} b_{2}^{2}+b_{2}^{2} b_{1}+b_{1} b_{2} b_{1}+1=0,
\end{aligned}
$$

and denote by $R \subset H(Q, 3)$ the span of $R_{0}, R_{1}, R_{2}$.

Lemma B.2. We have an isomorphism of vector spaces $I \cong R$.

Proof. We remark that

$$
b_{1} R_{0}=R_{0} b_{1}=R_{1} ; \quad b_{1} R_{1}=R_{1} b_{1}=R_{2} ; \quad b_{1} R_{2}=R_{2} b_{1}=R_{0} .
$$


Since $b_{j}^{3}=1$, we derive $b_{1} b_{2}^{2} b_{1}^{2}=b_{2}^{2} b_{1}^{2} b_{2}$. This implies also

$$
b_{2} R_{0}=R_{0} b_{2}=R_{1} ; \quad b_{2} R_{1}=R_{1} b_{2}=R_{2} ; \quad b_{2} R_{2}=R_{2} b_{2}=R_{0} .
$$

From these relations we find $x R_{0} y \in R$ for all $x, y \in H(Q, 3)$, hence $I \subset R$. The other inclusion is trivial.

Finally $K_{3}=H(Q, 3) / I$ will have dimension 21 , as wanted, since $\operatorname{dim}_{\mathbb{C}} R=3$. This ends the proof the proposition.

Acknowledgements. This paper is part of author's PhD thesis at University of Paris-Sud. Without the patience and the critical remarks of Pierre Vogel and Gregor Masbaum we would not arrive at the present version of this paper. I am indebted to my thesis advisor Valentin Poenaru for all discussions we had on this subject and the suggestions he gave me, and to Larry Siebenmann for his interest. I wish to thank Barbu Berceanu for having explained to me the method of Bergmann and for working with me for the huge computations from Appendix A, and the referee for the valuable comments improving the clarity of this paper.

\section{References}

[Bae92] Baez, J.C.: Link invariants of finite type and perturbation theory. Lett. Math. Phys. 26, 43-51 (1992)

[Ber78] Bergmann, G.M.: The diamond lemma for ring theory. Adv. in Math. 29, 178-218 (1978)

[Ber93] Berceanu, B.: Local homotopy of low dimensional varieties. Thesis, 1993

[Bir93] Birman, J.: New points of view in knot theory. Bull. A.M.S. 28, 253-287 (1993)

[Bou82] Bourbaki, N.: Groupes et algebres de Lie IV, V, VI. Paris: Masson, 1982

[BW89] Birman, J., Wenzl, H.: Braids, link polynomials and a new algebra. Trans. Am. Math. Soc. 313, 249-273 (1989)

[BXS93] Birman, J., Lin, X.-S.: Knot polynomials and Vassiliev's invariants. Invent. Math. 111, 225-270 (1993)

[Jon87] Jones, V.: Hecke algebras and a polynomial invariant for knots. Ann. Math. 126, 335-388 (1987)

Communicated by A. Connes 